\title{
Spatially-resolved dust properties of the GRB 980425 host galaxy ${ }^{\star \star \star}$
}

M. J. Michałowski ${ }^{1,2, \star \star \star}$, L. K. Hunt ${ }^{3}$, E. Palazzi ${ }^{4}$, S. Savaglio ${ }^{5}$, G. Gentile ${ }^{1,6}$, J. Rasmussen ${ }^{7,8}$, M. Baes ${ }^{1}$, S. Basa ${ }^{9}$, S. Bianchi ${ }^{3}$, S. Berta ${ }^{5}$, D. Burlon ${ }^{10}$, J. M. Castro Cerón ${ }^{11}$, S. Covino $^{12}$, J.-G. Cuby ${ }^{9}$, V. D’Elia ${ }^{13,14}$, P. Ferrero ${ }^{15,16}$, D. Götz ${ }^{17}$, J. Hjorth ${ }^{7}$, M. P. Koprowski ${ }^{2}$, D. Le Borgne ${ }^{18}$, E. Le Floc'h ${ }^{17}$, D. Malesani ${ }^{7}$, T. Murphy ${ }^{10}$, E. Pian ${ }^{4}$, S. Piranomonte ${ }^{19}$, A. Rossi ${ }^{20}$, J. Sollerman ${ }^{21}$, N. R. Tanvir ${ }^{22}$, A. de Ugarte Postigo ${ }^{23,7}$, D. Watson ${ }^{7}$, P. van der Werf ${ }^{24}$, S. D. Vergani ${ }^{12}$, and D. $\mathrm{Xu}^{7}$

1 Sterrenkundig Observatorium, Universiteit Gent, Krijgslaan 281-S9, 9000 Gent, Belgium e-mail: mm@roe.ac.uk

2 SUPA ${ }^{\star \star \star \star}$, Institute for Astronomy, University of Edinburgh, Royal Observatory, Edinburgh, EH9 3HJ, UK

3 INAF - Osservatorio Astrofisico di Arcetri, Largo E. Fermi 5, 50125 Firenze, Italy

4 INAF - IASF Bologna, via Gobetti 101, 40129 Bologna, Italy

5 Max-Planck-Institut für Extraterrestrische Physik, Giessenbachstraße, 85748 Garching bei München, Germany

${ }^{6}$ Department of Physics and Astrophysics, Vrije Universiteit Brussel, Pleinlaan 2, 1050 Brussels, Belgium

7 Dark Cosmology Centre, Niels Bohr Institute, University of Copenhagen, Juliane Maries Vej 30, 2100 Copenhagen $\varnothing$, Denmark

8 Technical University of Denmark, Department of Physics, Frederiksborgvej 399, 4000 Roskilde, Denmark

9 Aix Marseille Université, CNRS, LAM (Laboratoire d'Astrophysique de Marseille) UMR 7326, 13388 Marseille, France

10 Sydney Institute for Astronomy, School of Physics, The University of Sydney, Sydney NSW 2006, Australia

11 Herschel Science Centre (ESA-ESAC), 28.692 Villanueva de la Cañada, Madrid, Spain

12 INAF/Osservatorio Astronomico di Brera, via Emilio Bianchi 46, 23807 Merate (LC), Italy

13 ASI Science Data Centre, via Galileo Galilei, 00044 Frascati (RM), Italy

14 INAF - Osservatorio Astronomico di Roma, via di Frascati, 33, 00040 Monteporzio Catone, Italy

15 Instituto de Astrofísica de Canarias (IAC), 38200 La Laguna, Tenerife, Spain

16 Departamento de Astrofísica, Universidad de La Laguna (ULL), 38205 La Laguna, Tenerife, Spain

17 Laboratoire AIM-Paris-Saclay, CEA/DSM/Irfu - CNRS - Université Paris Diderot, CE-Saclay, pt courrier 131, 91191 Gif-sur-Yvette, France

18 Institut d'Astrophysique de Paris, UMR 7095, CNRS, UPMC Univ. Paris 06, 98bis boulevard Arago, 75014 Paris, France

19 INAF - Osservatorio Astronomico di Roma, via Frascati 33, 00040 Monte Porzio Catone (RM), Italy

20 Thüringer Landessternwarte Tautenburg, Sternwarte 5, 07778 Tautenburg, Germany

21 The Oskar Klein Centre, Department of Astronomy, AlbaNova, Stockholm University, 10691 Stockholm, Sweden

22 Department of Physics and Astronomy, University of Leicester, University Road, Leicester, LE1 7RH, UK

23 Instituto de Astrofísica de Andalucía (IAA-CSIC), Glorieta de la Astronomía s/n, 18008 Granada, Spain

${ }^{24}$ Leiden Observatory, Leiden University, PO Box 9513, 2300 RA Leiden, The Netherlands

Received 14 October 2013 / Accepted 25 November 2013

\section{ABSTRACT}

Gamma-ray bursts (GRBs) have been proposed as a tool for studying star formation in the Universe, so it is crucial to investigate whether their host galaxies and immediate environments are in any way special compared with other star-forming galaxies. Here we present spatially resolved maps of dust emission of the host galaxy of the closest known GRB 980425 at $z=0.0085$ using our new high-resolution observations from Herschel, Atacama Pathfinder Experiment (APEX), Atacama Large Millimeter Array (ALMA) and Australia Telescope Compact Array (ATCA). We modelled the spectral energy distributions of the host and of the star-forming region displaying the Wolf-Rayet signatures in the spectrum (WR region), located $800 \mathrm{pc}$ from the GRB position. The host is characterised by low dust content and a high fraction of UV-visible star formation, similar to other dwarf galaxies. These galaxies are abundant in the local universe, so it is not surprising to find a GRB in one of them, assuming the correspondence between the GRB rate and star formation. The WR region contributes substantially to the host emission at the far-infrared, millimetre, and radio wavelengths and we propose that this is a consequence of its high gas density. If dense environments are also found close to the positions of other GRBs, then the ISM density should also be considered, along with metallicity, an important factor influencing whether a given stellar population can produce a GRB.

Key words. dust, extinction - galaxies: individual: ESO 184-G82 - galaxies: star formation - submillimeter: galaxies gamma-ray burst: individual: 980425 - galaxies: ISM

\footnotetext{
* Herschel is an ESA space observatory with science instruments provided by European-led Principal Investigator consortia and with important participation from NASA.

$\star \star$ Reduced images as FITS files are only available at the CDS via anonymous ftp to cdsarc.u-strasbg.fr (130.79.128.5) or via http://cdsarc.u-strasbg.fr/viz-bin/qcat?J/A+A/562/A70

$\star \star \star$ FWO Pegasus Marie Curie Fellow.

$\star \star \star \star$ Scottish Universities Physics Alliance.
} 


\section{Introduction}

Long (duration >2 s) gamma-ray bursts (GRBs) have been shown to be associated with the death of massive stars (e.g. Hjorth et al. 2003; Stanek et al. 2003; for a review see Hjorth \& Bloom 2012), and therefore signal very recent star formation in their host galaxies. This is also supported by the detection of the signatures of Wolf-Rayet (WR) stars in GRB hosts (Hammer et al. 2006; Han et al. 2010; but see Chen et al. 2007; Schulze et al. 2011), by very low ages of the GRB sites (Christensen et al. 2008; Thöne et al. 2008; Östlin et al. 2008), and by the concentration of GRBs in the UV-brightest pixels of their hosts (Bloom et al. 2002; Fruchter et al. 2006; Kelly et al. 2008), similar to that of WR stars and supernovae Ic (Leloudas et al. 2010). Theoretically, rotating WR stars are the best candidates for long GRB progenitors, mainly because of their masses, angular momentum, and lack of the hydrogen envelope (Woosley 1993; Woosley \& Heger 2006; Yoon \& Langer 2005; Yoon et al. 2006). However, the debate about whether the GRB rate is proportional to the star formation rate (SFR) density in the Universe is still ongoing (Conselice et al. 2005; Castro Cerón et al. 2006; Fruchter et al. 2006; Stanek et al. 2006; Wainwright et al. 2007; Yüksel et al. 2008; Fynbo et al. 2009; Kistler et al. 2009; Savaglio et al. 2009; Leloudas et al. 2010, 2011; Levesque et al. 2010a,b; Svensson et al. 2010; Elliott et al. 2012; Hjorth et al. 2012; Michałowski et al. 2012b; Robertson \& Ellis 2012; Boissier et al. 2013; Hao \& Yuan 2013; Perley et al. 2013).

Since the presence of dust is intimately connected to star formation, we would expect to find measurable amounts of dust in GRB hosts. The characteristics of the dusty medium can be studied from its emission at long wavelengths, in particular in the far-infrared (far-IR) and submillimetre regimes that trace the emission of colder $(<60 \mathrm{~K})$ dust, which constitutes most of the total dust mass of a galaxy. However, because of insufficient sensitivity of previous instruments, only four GRB hosts have been detected at submillimetre wavelengths (Frail et al. 2002; Berger et al. 2003; Tanvir et al. 2004; Wang et al. 2012; see the full compilation of submillimetre observations in de Ugarte Postigo et al. 2012).

It is only now, with the successful operation of the Herschel Space Observatory (Pilbratt et al. 2010) and the Atacama Large Millimeter Array (ALMA), that the dust in a significant number of GRB hosts can be studied, as these facilities are capable of not only detecting its emission, but also mapping its spatial distribution. This is crucial in order to understand the interplay between the star formation giving rise to the GRB progenitor and the dust formation and to understand the physical conditions necessary for a massive star to explode as a GRB. It has been claimed that GRBs reside in low-metallicity environments, which could be reflected in the distribution of dust within their hosts.

Mapping the spatial distribution of dust is limited to the closest and most extended GRB hosts, so an obvious candidate for a resolved dust study is GRB 980425 at redshift $z=0.0085$ (Tinney et al. 1998) and its associated supernova SN 1998bw (Galama et al. 1998) located in the barred spiral galaxy ESO 184-G82. As this galaxy hosted the closest known GRB, it has been the subject of numerous studies (Fynbo et al. 2000; Sollerman et al. 2005; Foley et al. 2006; Hammer et al. 2006; Stanek et al. 2006; Hatsukade et al. 2007; Le Floc'h et al. 2006, 2012; Christensen et al. 2008; Michałowski et al. 2009, 2012b; Savaglio et al. 2009; Castro Cerón et al. 2010). It is a dwarf $\left(0.02 L_{B}^{*}\right)$ barred spiral showing a large number of starforming regions (Fynbo et al. 2000; Sollerman et al. 2005);
SN 1998bw occurred inside one of these (Fynbo et al. 2000; Sollerman et al. 2002), 800 pc southeast of a region displaying a Wolf-Rayet type spectrum (Hammer et al. 2006, hereafter: WR region). This region dominates the galaxy's emission at $24 \mu \mathrm{m}$ (Le Floc'h et al. 2006), contributes substantially to its SFR (but not to its stellar mass; Michałowski et al. 2009), is young (1-6 Myr; Hammer et al. 2006; Christensen et al. 2008), and exhibits the lowest metallicity among star-forming regions within the host with $12+\log (\mathrm{O} / \mathrm{H})=8.16$, i.e. 0.3 solar (Christensen et al. 2008) compared with $12+\log (\mathrm{O} / \mathrm{H})=8.6$, i.e. 0.8 solar (Sollerman et al. 2005) for the entire host (a common characteristic in nearby GRBs; Fig. 3 of Levesque et al. 2011; Thöne et al. 2008). Highly ionised mid-IR emission lines of the WR region also suggest a very young episode of star formation (Le Floc'h et al. 2012).

Because GRB 980425 ended its life in a region with very few massive stars which does not exhibit any WR spectral signatures, Hammer et al. (2006) have proposed that the GRB progenitor had been expelled from the nearby WR region with a velocity of the order of $\sim 100-300 \mathrm{~km} \mathrm{~s}^{-1}$, not atypical of Galactic runaway stars (Blaauw 1993; Tenjes et al. 2001; de Wit et al. 2005).

Even for this nearby and well studied GRB host the cold dust properties are not very well constrained, because the currently available infrared observations of this galaxy are limited to wavelengths shortward of $160 \mu \mathrm{m}$ (Le Floc'h et al. 2012). The objective of this paper is to change this situation by determining the dust properties in this closest known GRB host. This is possible with our new high-resolution data ranging from far-IR to radio.

This paper is structured as follows. In Sect. 2 we describe our data. Then, in Sect. 3, we describe the spectral energy distribution (SED) modelling we apply to these data, and present our results in Sect. 4. We discuss the implications of our results in Sect. 5, and close with a summary of our results in Sect. 6. We use a cosmological model with $H_{0}=70 \mathrm{~km} \mathrm{~s}^{-1} \mathrm{Mpc}^{-1}$, $\Omega_{\Lambda}=0.7$, and $\Omega_{\mathrm{m}}=0.3$, so GRB 980425 at $z=0.0085$ is at a luminosity distance of $36.5 \mathrm{Mpc}$ and $1^{\prime \prime}$ corresponds to $175 \mathrm{pc}$ at its redshift (i.e. our resolution of $\sim 7^{\prime \prime}$ at $100 \mu \mathrm{m}$ corresponds to $1.3 \mathrm{kpc})$.

\section{Data}

We obtained Herschel observations of the GRB 980425 host (project no. OT1_lhunt_2, PI: L. Hunt) using the Photodetector Array Camera and Spectrometer (PACS; Poglitsch et al. 2010) and the Spectral and Photometric Imaging Receiver (SPIRE; Griffin et al. 2010) with a total integration time of 1116 and $445 \mathrm{~s}$, respectively, on 13-14 Mar 2011 $1^{1}$. The Herschel observations of the entire GRB host sample within this program are presented in Hunt et al. (2014a).

We used PACS in Small-Scan map mode $\left(20^{\prime \prime} / \mathrm{s}\right)$, with ten scan legs, $3^{\prime}$ long, separated by $4^{\prime \prime}$ steps. The scans were divided into two astronomical observation requests (AORs), with orthogonal scan directions that were executed sequentially. With this configuration we obtained homogeneous coverage over an area with a diameter of $\sim 2^{\prime}$, sufficient to cover the region subtended by the GRB 980425 host galaxy. Cross scans gave the needed redundancy to avoid $1 / f$ noise and spurious detector glitches on science and noise maps. Our estimated $1 \sigma$ sensitivity is 0.9 and $2.1 \mathrm{mJy}$ at 100 and $160 \mu \mathrm{m}$, respectively. With SPIRE in Small-Map Mode, we used three repetitions in order to obtain a

OBSIDs: 1342216005,1342216055 and 1342216056. 
sensitivity of roughly the $1 \sigma$ confusion limit (see Nguyen et al. 2010) of $\sim 6 \mathrm{mJy}^{-1}$ beam $^{-1}$ at $250 \mu \mathrm{m}$.

Data reduction for PACS and SPIRE was performed with Herschel imaging processing environment (HIPE; Ott 2010) v10.0. For PACS, we used HIPE to obtain the initial calibration and reduction, and then scanamorphos (v16.0; Roussel 2013) to make the final maps, in order to better account for faint, extended emission. We used pixel sizes of 1'.7 and 3.'00 for PACS 100 and $160 \mu \mathrm{m}$, and 4.'5, 6.'25, and 9.'0 for SPIRE 250, 350 , and $500 \mu \mathrm{m}$, respectively.

In each PACS and SPIRE image, an estimate for the background was obtained by averaging the flux measured within a set of sky apertures close to the galaxy but far enough away to avoid the contamination from the galaxy emission.

After background subtraction, the flux densities of the entire host galaxy at all Herschel wavelengths were obtained in an aperture of 50" diameter. The aperture corrections were negligible in all cases when compared with calibration uncertainties (Ciesla et al. 2012; Dale et al. 2012).

Because of limitations in image resolution it was possible to measure reliably the WR region flux only for the PACS data at $100 \mu \mathrm{m}$ and $160 \mu \mathrm{m}$ where the source is point-like. An aperture with a radius of $6^{\prime \prime}$ was used to measure the flux and a standard PSF extrapolation was applied for aperture correction.

The uncertainties associated with the measured photometric values reported in Table 1 were computed as a combination in quadrature of the calibration uncertainty, $7 \%$ for SPIRE data (according to Version 2.4, 7 June 2011, of the SPIRE Observer's Manual) and 5\% for PACS data (according to Version 2.3, 8 June 2011 of the PACS Observer's Manual), and the sky uncertainty derived by considering the number of pixels within the galaxy and the WR region apertures and the standard deviation of the average value in the individual sky apertures (see Dale et al. 2012). Before addition in quadrature, the sky uncertainties were corrected for the correlated noise, since the pixels are not independent.

We also performed submillimetre $(870 \mu \mathrm{m})$ observations of the GRB 980425 host on 4-5 Aug. 2007 (project no. O-079.F-9312A-2007, PI: M. Michałowski) using the Large Apex BOlometer CAmera (LABOCA; Siringo et al. 2009) mounted at the Atacama Pathfinder Experiment (APEX; Güsten et al. 2006). A total of $4.1 \mathrm{~h}$ of on-source data were obtained. During most of the observations the weather was excellent with $0.25 \mathrm{~mm}$ of precipitable water vapour. Data reduction was done using the CRUSH ${ }^{2}$ package (Kovács 2008). We used the "deep" option that results in the best signal-to-noise ratio for faint objects. We checked that the "faint" option, with weaker filtering, resulted in a higher noise level. The analysis was done using the MIRIAD package (Sault \& Killeen 2004; Sault et al. 1995). The beam size for the final image was 19.5 and the rms was $1.4 \mathrm{mJy}_{\text {beam }}{ }^{-1}$. The target was detected and is extended at the resolution of APEX/LABOCA with the measured full width at half maximum $(\mathrm{FWHM})$ of $(35 \pm 11)^{\prime \prime} \times(30 \pm 9)^{\prime \prime}$, and deconvolved size of $29^{\prime \prime} \times 23^{\prime \prime}$, consistent with the size at optical wavelengths (half-light diameter at $R$-band of $22^{\prime \prime}$, i.e. $4 \mathrm{kpc}$ ). The flux for the entire galaxy was found by summing the signal over a $50^{\prime \prime}$ diameter aperture. The resolution of the data was not sufficient to separate the WR region from the rest of the galaxy.

We performed Band 3 ALMA observations on 1 Sep. 2012 (project no. 2011.0.00046.S, PI: M. Michałowski). A total of $67.4 \mathrm{~min}$ of on-source data were obtained. Four $1.875 \mathrm{GHz}$

\footnotetext{
www. submm.caltech.edu/ sharc/crush/
}

Table 1. Our new UVOT, WISE, Herschel/PACS and SPIRE, APEX/ LABOCA, ALMA and ATCA photometry results, the contribution of the WR region and the beam sizes. The limit is $2 \sigma$.

\begin{tabular}{lccccc}
\hline \hline $\begin{array}{l}\lambda_{\text {obs }} \\
(\mu \mathrm{m})\end{array}$ & $\begin{array}{c}\text { Entire host } \\
(\mathrm{mJy})\end{array}$ & $\begin{array}{c}\text { WR region } \\
(\mathrm{mJy})\end{array}$ & $\%$ & $\begin{array}{c}F W H M \\
\left({ }^{\prime \prime}\right)\end{array}$ & Inst. \\
\hline 0.2033 & $1.70 \pm 0.05$ & $0.15 \pm 0.01$ & 9 & 2.92 & UVOT \\
0.2229 & $1.70 \pm 0.05$ & $0.15 \pm 0.01$ & 9 & 2.45 & UVOT \\
0.2591 & $1.79 \pm 0.06$ & $0.14 \pm 0.01$ & 8 & 2.37 & UVOT \\
3.4 & $4.3 \pm 0.3$ & $0.54 \pm 0.04$ & 1.3 & 6.1 & WISE \\
4.6 & $2.3 \pm 0.3$ & $0.32 \pm 0.03$ & 14 & 6.4 & WISE \\
12 & $11.9 \pm 0.8$ & $2.1 \pm 0.1$ & 18 & 6.5 & WISE \\
22 & $28.7 \pm 0.9$ & $16 \pm 1$ & 56 & 12 & WISE \\
100 & $327 \pm 12$ & $72 \pm 5$ & 19 & $6.7 \times 6.9$ & PACS \\
160 & $323 \pm 19$ & $74 \pm 9$ & 13 & $10.7 \times 12.1$ & PACS \\
250 & $231 \pm 16$ & $\cdots$ & $\cdots$ & 18.2 & SPIRE \\
350 & $106 \pm 8$ & $\cdots$ & $\cdots$ & 24.9 & SPIRE \\
500 & $43 \pm 4$ & $\cdots$ & $\cdots$ & 36.3 & SPIRE \\
870 & $12.7 \pm 1.8$ & $\cdots$ & $\cdots$ & 19.5 & APEX \\
2790.5 & $<0.43$ & $0.123 \pm 0.025$ & $>29$ & $1.9 \times 1.8$ & ALMA \\
2790.5 & $\cdots$ & $\cdots .069^{a} \pm 0.028$ & $\cdots$ & $1.9 \times 1.8$ & ALMA \\
$1.05 \mathrm{e} 5$ & $0.40 \pm 0.20$ & $0.28 \pm 0.03$ & 70 & $10.3 \times 7.0$ & ATCA \\
$1.28 \mathrm{e} 5$ & $0.71 \pm 0.19$ & $0.29 \pm 0.03$ & 41 & $12.0 \times 7.5$ & ATCA \\
$1.63 \mathrm{e} 5$ & $1.11 \pm 0.21$ & $0.42 \pm 0.04$ & 38 & $13.5 \times 8.3$ & ATCA \\
$2.26 \mathrm{e} 5$ & $0.84 \pm 0.16$ & $0.60 \pm 0.06$ & 71 & $23 \times 14$ & ATCA \\
\hline
\end{tabular}

Notes. ${ }^{(a)}$ With free-free emission subtracted as described in Sect. 3.

spectral windows were centred at 100.6, 102.4, 112.5, and $114.3 \mathrm{GHz}$. Twenty three antennas and baselines ranging between 24 and $384 \mathrm{~m}$ were available. Neptune, J1733-130, and J1945-552 were used as flux, bandpass, and phase calibrators, respectively. The amount of precipitable water vapour ranged between 1.8-2.15 mm. The data reduction and analysis were done using the CASA package (McMullin et al. 2007). The map was created by multi-frequency synthesis with "natural" weighting, giving the best signal-to-noise ratio. The WR region is the only detected star-forming region in the GRB 980425 host. We measured its flux by fitting a Gaussian at its position, whereas the flux for the entire galaxy was again measured in the $50^{\prime \prime}$ diameter aperture.

We performed radio $2.1 \mathrm{GHz}$ observations with the Australia Telescope Compact Array (ATCA) on 12 Apr. 2012 with a total integration time of $12 \mathrm{~h}$ (project no. C2700, PI: M. Michałowski). The array was in the $1.5 \mathrm{~B}$ configuration with baselines up to $1500 \mathrm{~m}$. We used $2 \mathrm{GHz}$ bandwidth. The data reduction and analysis were done using MIRIAD. We analysed the data separately in four $0.5 \mathrm{GHz}$ frequency ranges centred at $1324,1836,2348$, and $2860 \mathrm{MHz}$. The WR region is detected at all four of these frequencies dominating the radio flux of the GRB 980425 host. Again, we used a Gaussian fit and a 50" diameter aperture to measure the flux of the WR region and the entire galaxy, respectively.

We also obtained Ultraviolet/Optical Telescope (UVOT; Roming et al. 2005) data from the Swift (Gehrels et al. 2004) archive $^{3}$. The GRB 980425 host was observed on 15-16 Jun. 2007 (obsid 00036585001) and on 18 Jun 2007 (obsid 00036585002) with total integration times of 2034, 1350, and $1014 \mathrm{~s}$ for the $u v w 2(0.20 \mu \mathrm{m}), u v m 2(0.22 \mu \mathrm{m})$, and $u v w 1$ $(0.26 \mu \mathrm{m})$ filters, respectively. We only used these filters, as the data at other (optical) filters are superseded by the groundbased data. We measured the fluxes of the entire galaxy in

\footnotetext{
3 http://heasarc.gsfc.nasa.gov/cgi-bin/W3Browse/ swift.pl
} 
a 50" aperture and derived the aperture corrections from the curve-of-growth analysis. For the WR region we used a standard aperture with a radius of $5^{\prime \prime}$. We added in quadrature 0.03 mag to the errors to account for zero-point uncertainty ${ }^{4}$. We corrected the fluxes for Galactic extinction assuming $E(B-V)=$ 0.059 mag (Schlegel et al. 1998; Sollerman et al. 2005) and the extinction curve of Cardelli et al. (1989). The photometry is fully consistent with lower resolution GALEX data (Michałowski et al. 2009).

Finally, we used the Wide-field Infrared Survey Explorer (WISE) All-Sky images ${ }^{5}$ (Wright et al. 2010). Again, for the entire galaxy we used a 50" aperture and the curve-of-growth analysis. For the WR region we used standard apertures (radius 8.25" for band 1-3 and 16.25" for band 4) and applied appropriate aperture corrections ${ }^{6}$. Our fluxes are consistent with those from Spitzer (Le Floc'h et al. 2006) for overlapping filters.

The UVOT, WISE, Herschel, LABOCA, ALMA, and ATCA images are shown in Fig. 1 and the results of the photometry are summarised in Table 1. A shift in the flux centroid to the west from $3.4 \mu \mathrm{m}$ to $22 \mu \mathrm{m}$ and to the east from $160 \mu \mathrm{m}$ to $500 \mu \mathrm{m}$ is a result of the increasing (Le Floc'h et al. 2006) and decreasing contribution of the WR region to the total flux, respectively.

For the SED modelling we also used the compilation of the data presented in Michałowski et al. (2009, their Table 1) covering ultraviolet (UV) to radio wavelengths (including the data from Watson et al. 2004; Sollerman et al. 2005; Le Floc'h et al. 2006; Castro Cerón et al. 2010). We also used the $70 \mu \mathrm{m}$ photometry of the entire host from Le Floc'h et al. (2012), but not at $160 \mu \mathrm{m}$, as these data are superseded by our higher-resolution and more sensitive data. Their measurements are consistent with ours within the uncertainties.

\section{SED fitting}

Before fitting the SED, we estimated the free-free emission (Caplan \& Deharveng 1986) in the WR region using the H $\alpha$ flux measured by Christensen et al. (2008) corrected for Galactic extinction. The estimated free-free flux at $2.8 \mathrm{~mm}$ is $0.054 \mathrm{mJy}$, $\sim 45 \%$ of the total observed WR flux. Hence, we used in the fit at $2.8 \mathrm{~mm} 0.069 \mathrm{mJy}$, which is the remainder after correcting for the free-free emission. The $2.8 \mathrm{~mm}$ free-free emission over the entire galaxy is found to be $\sim 0.21 \mathrm{mJy}$, well under the upper limit of our non-detection. At $870 \mu \mathrm{m}$ we estimate a total free-free contribution of $\sim 0.19 \mathrm{mJy}$, less than $2 \%$ of the total observed flux and smaller than the calibration uncertainties; hence we neglect its correction at this wavelength.

We applied the SED fitting method detailed in Michałowski et al. (2008, 2009, 2010a,c, see therein a discussion of the derivation of galaxy properties and typical uncertainties) based on 35000 templates in the library of Iglesias-Páramo et al. (2007), plus additional templates of Silva et al. (1998) and Michałowski et al. (2008), all developed in GRASIL ${ }^{7}$ (Silva et al. 1998). They are based on numerical calculations of radiative transfer within a galaxy that is assumed to be a triaxial system with diffuse dust and dense molecular clouds, in which stars are born.

The templates cover a broad range of galaxy properties from quiescent to starburst. Their star formation histories are assumed

\footnotetext{
4 http://heasarc.gsfc.nasa.gov/docs/swift/analysis/ uvot_digest/zeropts.html.

5 http://wise.ssl. berkeley.edu/astronomers.html.

6 http://wise2.ipac.caltech.edu/docs/release/allsky/ expsup/

7 www.adlibitum.oat.ts.astro.it/silva
}

to represent a smooth Schmidt-type law (SFR proportional to the gas mass to some power; see Silva et al. 1998, for details) with a starburst (if any) in addition starting 50 Myr before the time at which the SED is computed. There are seven free parameters in the library of Iglesias-Páramo et al. (2007): the normalization of the Schmidt-type law, the timescale of the mass infall, the intensity of the starburst, the timescale of the molecular cloud destruction, the optical depth of molecular clouds, the age of a galaxy, and the inclination of a disk with respect to the observer.

We also used MAGPHYs ${ }^{8}$ (Multi-wavelength Analysis of Galaxy Physical Properties; da Cunha et al. 2008), an empirical but physically-motived SED model, which is based on the energy balance between the energy absorbed by dust and reemitted in the infrared. We used the Bruzual \& Charlot (2003) stellar models and adopted the Chabrier (2003) initial mass function (IMF). Similarly to GRASIL, in MAGPHYS two dust media are assumed: diffuse interstellar medium (ISM) and dense stellar birth clouds. Four dust components are considered: cold dust (15-25 K), warm dust (30-60 K), hot dust (130-250 K), and polycyclic aromatic hydrocarbons (PAHs).

\section{Results}

\subsection{Basic physical properties}

The best-fit SEDs $^{9}$ are shown in Fig. 2 and the parameters derived from the MAGPHYS fit are shown in Table 2. No GRASIL model was found to have sufficiently high UV emission and relatively low far-IR emission to explain the data for the entire host. However, for the WR region the GRASIL model found by Michałowski et al. (2009) fits our new photometry as well. The WR datapoints at $\sim 0.4-0.6 \mu \mathrm{m}$ are underestimated by the models, but this spectral range is dominated by strong emission lines (Hammer et al. 2006; Christensen et al. 2008) not accounted for in the models.

With the excellent wavelength coverage at the dust peak, we confirm that the WR region contributes $25 \%$ to the infrared luminosity of the host (cf. Sollerman et al. 2005; Le Floc'h et al. 2006, 2012; Christensen et al. 2008; Michałowski et al. 2009). However, for the first time we were able to constrain its dust content and we found that it contributes only 5\% to the dust mass of the host. Its lower contribution to the dust mass compared with infrared luminosity is a consequence of its high dust temperature (both dust components in the WR region are hotter than those in the entire host, and the contribution of the hotter component is higher in the WR region, see Cols. 8-11 in Table 2). As in Michałowski et al. (2009) we found that the WR region contributes very little to the stellar mass of the host.

We also performed SED modelling using the photometry of the host after subtracting the WR region contribution (green line in Fig. 2 and the last row in Table 2). Given the strong contribution of the WR region only in the mid-IR, it is unsurprising that the properties we derived from this "host-WR" photometry are very similar to those obtained using the original host photometry, except for much weaker contribution of hot $T_{\mathrm{d}}>40 \mathrm{~K}$ dust to the infrared luminosity, as the "warm" component has only $\sim 35 \mathrm{~K}$ for the "host-WR" photometry (see Col. 10 in Table 2). Hence, a possible contribution of WR regions in higher redshift GRBs, which cannot be separated from host galaxies, should not strongly affect the global host properties. The stellar mass derived from the "host-WR" photometry is nominally

\footnotetext{
8 wwW .iap.fr/magphys

9 The SED fits can be downloaded from http://dark.nbi.ku.dk/ research/archive
} 

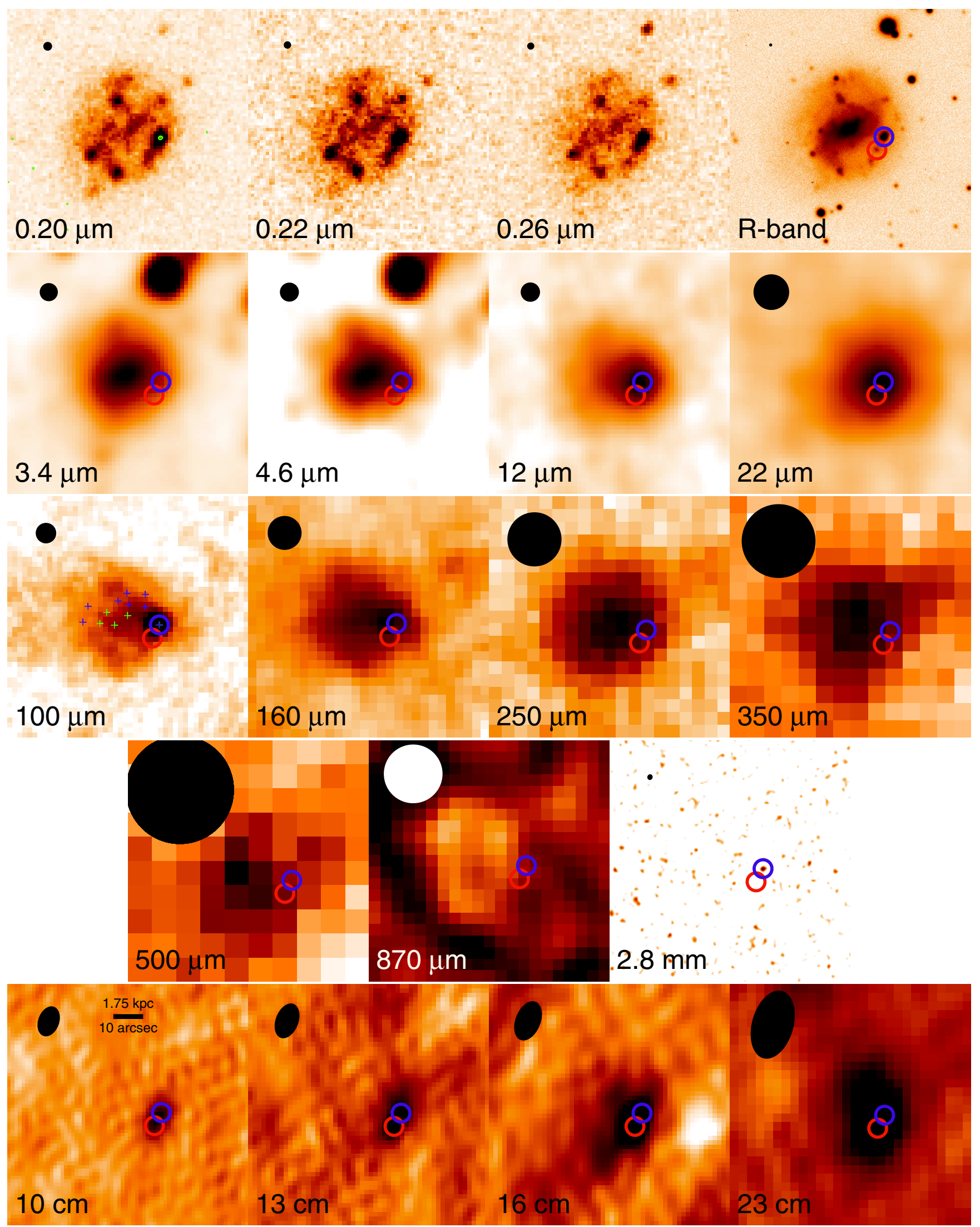

Fig. 1. Mosaic of the images of the GRB 980425 host. The $R$-band is from Sollerman et al. (2005) and the remaining images are presented here for the first time. North is up and east is to the left. Each panel is $80^{\prime \prime} \times 80^{\prime \prime}(14 \mathrm{kpc} \times 14 \mathrm{kpc})$. The scale is indicated on the $10 \mathrm{~cm}$ image, whereas the FWHM beamsize is shown on each panel as a filled circle or ellipse. The red and blue circles show the GRB position and the WR region, respectively. Blue and green crosses on the $100 \mu \mathrm{m}$ image show the star-forming regions with high electron density $n_{\mathrm{e}}>145 \mathrm{~cm}^{-3}$ and age $\geq 6 \mathrm{Myr}$, respectively (see Sect. 5.2). The ALMA $2.8 \mathrm{~mm}$ image is shown as 3.5, 4, and $5 \sigma$ contours on the $0.2 \mu \mathrm{m}$ image. The $870 \mu \mathrm{m}$ image has been inverted. 


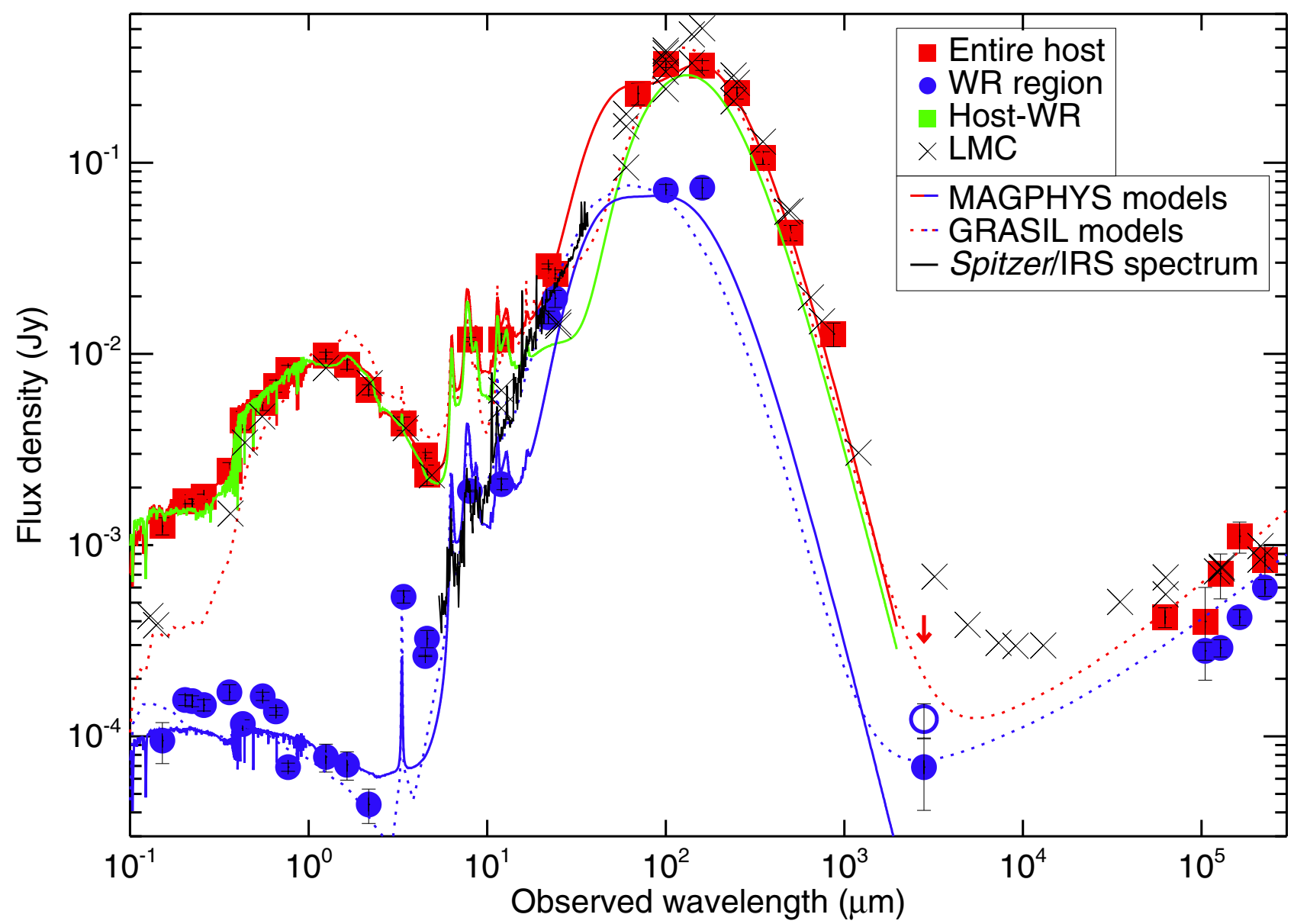

Fig. 2. Spectral energy distribution of the GRB 980425 host (red), the WR region (blue), and the host with the WR region subtracted (green). The data are shown as squares and circles, whereas the MAGPHYS and GRASIL models are shown as solid and dotted lines, respectively. The empty blue circle denotes the ALMA flux of the WR region without the correction for the free-free emission. The mid-IR Spitzer/IRS spectrum of the WR region (Le Floc'h et al. 2012) is shown as a black line. The "host-WR" photometry almost overlaps with the total host photometry in most cases, so it is not shown. The data for the LMC are shown as black crosses (Israel et al. 2010; Meixner et al. 2013).

Table 2. MAGPHYS results from the SED fitting.

\begin{tabular}{lcccccccccccccc}
\hline \hline Region & $\log L_{\mathrm{IR}}$ & $S F R$ & $s S F R$ & $\log M_{*}$ & $\log M_{\mathrm{d}}$ & $\tau_{V}$ & $T_{\text {cold }}$ & $\xi_{\text {cold }}$ & $T_{\text {warm }}$ & $\xi_{\text {warm }}$ & $\xi_{\text {hot }}$ & $\xi_{\text {PAH }}$ & $f_{\mu}$ \\
& $\left(L_{\odot}\right)$ & $\left(M_{\odot} \mathrm{yr}^{-1}\right)$ & $\left(\mathrm{Gyr}^{-1}\right)$ & $\left(M_{\odot}\right)$ & $\left(M_{\odot}\right)$ & & $(\mathrm{K})$ & & $(\mathrm{K})$ & & & & \\
$(1)$ & $(2)$ & $(3)$ & $(4)$ & $(5)$ & $(6)$ & $(7)$ & $(8)$ & $(9)$ & $(10)$ & $(11)$ & $(12)$ & $(13)$ & $(14)$ \\
\hline Entire host & $9.012_{-0.005}^{+0.005}$ & $0.26_{-0.08}^{+0.08}$ & 0.53 & $8.68_{-0.30}^{+0.30}$ & $6.21_{-0.01}^{+0.02}$ & 0.16 & $17.2_{-0.0}^{+0.8}$ & $0.22_{-0.01}^{+0.05}$ & $52_{-1}^{+4}$ & $0.53_{-0.01}^{+0.04}$ & $0.14_{-0.03}^{+0.00}$ & $0.082_{-0.000}^{+0.012}$ & $0.30_{-0.02}^{+0.02}$ \\
WR region & $8.407_{-0.000}^{+0.015}$ & $0.02_{-0.01}^{+0.01}$ & 2.37 & $7.00_{-0.30}^{+0.30}$ & $4.93_{-0.25}^{+0.29}$ & 0.47 & $21.7_{-2.7}^{+1.5}$ & $0.18_{-0.05}^{+0.00}$ & $60_{-1}^{+1}$ & $0.67_{-0.01}^{+0.05}$ & $0.09_{-0.01}^{+0.00}$ & $0.071_{-0.005}^{+0.000}$ & $0.19_{-0.03}^{+0.01}$ \\
$\%$ & 25 & 7 & $\ldots$ & 2 & 5 & $\ldots$ & $\cdots$ & $\cdots$ & $\cdots$ & $\cdots$ & $\cdots$ & $\cdots$ & $\cdots$ \\
Host-WR & $8.772_{-0.005}^{+0.005}$ & $0.19_{-0.06}^{+0.06}$ & 0.27 & $8.82_{-0.30}^{+0.30}$ & $5.84_{-0.26}^{+0.25}$ & 0.10 & $18.9_{-2.3}^{+2.6}$ & $0.24_{-0.07}^{+0.08}$ & $35_{-3}^{+5}$ & $0.47_{-0.08}^{+0.07}$ & $0.16_{-0.02}^{+0.02}$ & $0.129_{-0.010}^{+0.011}$ & $0.36_{-0.05}^{+0.05}$ \\
\hline
\end{tabular}

Notes. (1) The entire host galaxy/only the WR region/percentage contribution of the WR region to the cumulative properties of the galaxy/the host with WR fluxes subtracted. (2) $8-1000 \mu \mathrm{m}$ infrared luminosity. (3) Star formation rate from SED modelling. (4) Specific star formation rate $\left(\equiv S F R / M_{*}\right)$. (5) Stellar mass. (6) Dust mass. (7) Average $V$-band optical depth $\left(A_{V}=1.086 \tau_{V}\right)$. (8) Temperature of the cold dust component. (9) Contribution of the cold component to the infrared luminosity. (10) Temperature of the warm dust component. (11) Contribution of the warm component to the infrared luminosity. (12) Contribution of the hot (130-250 K, mid-IR continuum) component to the infrared luminosity. (13) Contribution of the PAH component to the infrared luminosity. (14) Contribution of the ISM dust (as opposed to birth clouds) to the infrared luminosity.

higher than that from the "entire host" photometry (though consistent within errors) because without the WR region the SED modelling converges to a slightly less active star-forming system with a higher mass-to-light ratio.

Our stellar mass for the entire host is $\sim 2$ times lower than that derived by Michałowski et al. (2009) and Savaglio et al. (2009) and 30\% lower than that derived by Castro Cerón et al. (2010), after correcting for the different IMF used. These differences are, however, comparable to the expected uncertainty of stellar mass estimates (see discussion in Michałowski et al. 2012a). Indeed, in contrast, our mass estimates for the WR region is a few times higher than that of Michałowski et al. (2009). The 
derived masses correspond to stellar mass-to-light ratios in the $H$ - and $K$-bands of $\sim 0.6$ and $\sim 1 M_{\odot} L_{\odot}^{-1}$ for the entire host, respectively and $\sim 1.5$ and $\sim 2.2 M_{\odot} L_{\odot}^{-1}$ for the WR region.

When we fitted a modified black body to the $100-870 \mu \mathrm{m}$ data for the entire host, we obtained $T_{\mathrm{d}} \sim 24 \pm 1 \mathrm{~K}$ assuming $\beta=1.5$, and $T_{\mathrm{d}} \sim 28 \pm 1 \mathrm{~K}$ assuming $\beta=1$ (which provides a better fit to the $870 \mu \mathrm{m}$ data). These temperatures are typical for its $L_{\mathrm{IR}}$; using the half-light radius of $r=4 \mathrm{kpc}$ we derived the infrared surface brightness $\Sigma_{\mathrm{IR}}=L_{\mathrm{IR}} / 2 \pi r_{h}^{2}=10 L_{\odot} \mathrm{pc}^{-2}$ (Eq. (2) of Chanial et al. 2007), which should indeed yield $T_{\mathrm{d}} \sim 26 \mathrm{~K}$ according to Eq. (23) of Chanial et al. (2007), which characterises the $T_{\mathrm{d}}-\Sigma_{\text {IR }}$ relation of local IRAS galaxies.

The dust mass estimates from MAGPHYs are based on the dust mass absorption coefficient $\kappa(850 \mu \mathrm{m})=0.77 \mathrm{~cm}^{2} \mathrm{~g}^{-1}$ (da Cunha et al. 2008; Dunne et al. 2000), which is approximately a factor of two higher than is assumed in the model of Draine \& Li (2007). Since such lower coefficient was applied to the local galaxies observed with Herschel, used in Sect. 5.1 as a comparison sample, we recomputed the dust mass of the GRB 980425 host applying a comparable method outlined in Bianchi (2013), obtaining $\log \left(M_{\mathrm{d}} / M_{\odot}\right)=6.57 \pm 0.05$, indeed a factor of two higher than the MAGPHYS estimate.

\subsection{Flux ratios}

In order to investigate the properties of the GRB 980425 host, in Fig. 3 we compared it with other samples of local galaxies with respect to $100 \mu \mathrm{m}, K$-band, and near-ultraviolet (NUV) flux ratios, characterizing specific star formation rate $\left(s S F R \equiv S F R / M_{*}\right.$ approximated by the $100 \mu \mathrm{m} / K$-band flux ratio), dust attenuation $(100 \mu \mathrm{m} / \mathrm{NUV})$ and near-infrared/UV colour $(K$-band/NUV). We also added the GRB 031203 host at $z=0.105$ using the data from Watson et al. (2011).

For comparison, we used the sample of Moustakas \& Kennicutt (2006), which includes UV-excess galaxies (comprising blue compact galaxies with strong emission lines, starburst nuclei, and normal massive spiral and irregular galaxies with abnormally high star formation rates), IRAS-selected galaxies, $\mathrm{H} \alpha$ - and UV-selected galaxies within $11 \mathrm{Mpc}$, star-forming galaxies in the Ursa Major Cluster and morphologically disturbed galaxies. Moreover, we used the sample of UV-selected galaxies of Gil de Paz et al. (2007), for which irregular galaxies are marked as green triangles in Fig. 3. We also used the sample of irregular galaxies and blue compact dwarfs (BCDs) from Hunt et al. (2005), Hirashita et al. (2008), and Hunter et al. (2010), for which we compiled the UV (Kinney et al. 1993; Dale et al. 2007; Gil de Paz et al. 2007), K-band (Thuan 1983; Jarrett et al. 2003; Hunt et al. 2005; Engelbracht et al. 2008), and $100 \mu \mathrm{m}$ photometry (Rice et al. 1988; Soifer et al. 1989; Sanders et al. 2003; Lisenfeld et al. 2007; Hirashita et al. 2008; Dale et al. 2009; Hunter et al. 2010). The Key Insights on Nearby Galaxies: a FarInfrared Survey with Herschel (KINGFISH) sample (Kennicutt et al. 2011; Dale et al. 2007, 2012; Skibba et al. 2011) is also used as a comparison; KINGFISH irregular galaxies are marked as green triangles in Fig. 3. Dust masses for the KINGFISH galaxies are taken from Bianchi (2013), and stellar masses from Skibba et al. (2011) are corrected to the Kennicutt et al. (2011) distance scale. Finally, we mark the Large Magellanic Cloud (LMC) using the compilation in Israel et al. (2010) and Meixner et al. (2013).

Both GRB 980425 and 031203 hosts exhibit low $100 \mu \mathrm{m} /$ NUV ratios (indicating low attenuation and dust content), within the range spanned by other local galaxies, especially late types and irregulars. Moreover, they have low $100 \mu \mathrm{m} / K$-band ratios (proxy for sSFR) compared with other galaxies, given their low $K$-band/NUV ratios, again similarly to late-type spirals.

In order to investigate its low $100 \mu \mathrm{m} / \mathrm{NUV}$ ratio we constructed the flux ratio map, in a similar way to that described by Boquien et al. $(2011,2012)$ : we converted both maps to Jy per pixel, subtracted the local sky background, corrected the NUV map for the extinction in our Galaxy using the parametrization of Cardelli et al. (1989) and O'Donnell (1994) with $E(B-V)=0.059 \mathrm{mag}$, and regridded the $100 \mu \mathrm{m}$ map to the NUV pixelscale. Then the $100 \mu \mathrm{m}$ and NUV maps were adaptively smoothed to the same spatial scales using ASMOOTH (Ebeling et al. 2006) to avoid strong colour fluctuations, and divided. The result is shown in Fig. 4 with the narrow-band $\mathrm{H} \alpha$ and $R$-band contours (Sollerman et al. 2005) overplotted. The former was confirmed to be consistent with the integral field unit (IFU) $\mathrm{H} \alpha$ spectroscopy from Christensen et al. (2008) in the part of the host covered by the IFU observations.

From this map we found that the regions with low $100 \mu \mathrm{m} /$ NUV ratios correspond closely to the star-forming regions dominating the $\mathrm{H} \alpha$ (and hence the SFR) image, with the exception of the WR region exhibiting very high $100 \mu \mathrm{m}$ flux.

\section{Discussion}

\subsection{Nature of the host: star-forming dwarf}

In terms of the basic properties, it has been suggested that the host of GRB 980425 is a typical dwarf galaxy (Sollerman et al. 2005; Christensen et al. 2008; Michałowski et al. 2009). Its SFR, stellar mass, size, and metallicity agree perfectly with that of the largest dwarf irregulars in the sample of Woo et al. (2008). Moreover, we also found it similar to irregular galaxies in terms of far-IR/UV flux ratios (Fig. 3). Finally, its sSFR is consistent with the high-end of samples of local galaxies with similar stellar masses (e.g. Gilbank et al. 2011; Gavazzi et al. 2013). The metallicity of the GRB 980425 of $12+\log (\mathrm{O} / \mathrm{H})=8.6$ (Sollerman et al. 2005) is roughly consistent with the typical value for a $10^{9} M_{\odot}$ galaxy in the mass-metallicity relations of Kewley \& Ellison (2008), and with the mass-metallicity relation of local dwarf galaxies (Hunt et al. 2012).

We also found that the GRB 980425 host has relatively low dust content, similar to local dwarf galaxies. Specifically, it exhibits a low $100 \mu \mathrm{m} / \mathrm{NUV}$ flux ratio (Fig. 3) and a dust-to-stellar ratio $\log \left(M_{\mathrm{d}} / M_{*}\right) \sim-2.1$ (using $M_{\mathrm{d}}$ derived in Sect. 4.1), consistent with that of the dwarf local galaxies in the HRS (Fig. 3 of Cortese et al. 2012) and KINGFISH surveys. It is also consistent with the low-end of the local $M_{\mathrm{d}}-\mathrm{SFR}$ relation (da Cunha et al. 2010). In Fig. 5 we also show that the GRB 980425 host has a very blue optical/UV colour and the dust content expected for such galaxies based on the HRS and KINGFISH surveys. Moreover, the dust-obscured SFR derived from the infrared luminosity of the host $\left(\mathrm{SFR}_{\mathrm{IR}}\right)$ is only $\sim 0.1 M_{\odot} \mathrm{yr}^{-1}$ (using the relation of Kennicutt 1998, converted to the Chabrier IMF), which is 2.5 times lower than the total SFR from the SED modelling (Table 2) and than the UV-only estimate (Michałowski et al. 2009). This means that most of its star formation activity is not dust-obscured. Finally, we demonstrate in Fig. 2 that although the SEDs of the GRB 980425 host and of the LMC are remarkably similar in the far-IR regime, the former is much brighter in the UV.

To summarise, the properties of the GRB 980425 host are consistent with those of a population of local dwarf irregular galaxies, which exhibit relatively low dust content and a high fraction of UV-visible star formation. The IRAS infrared 


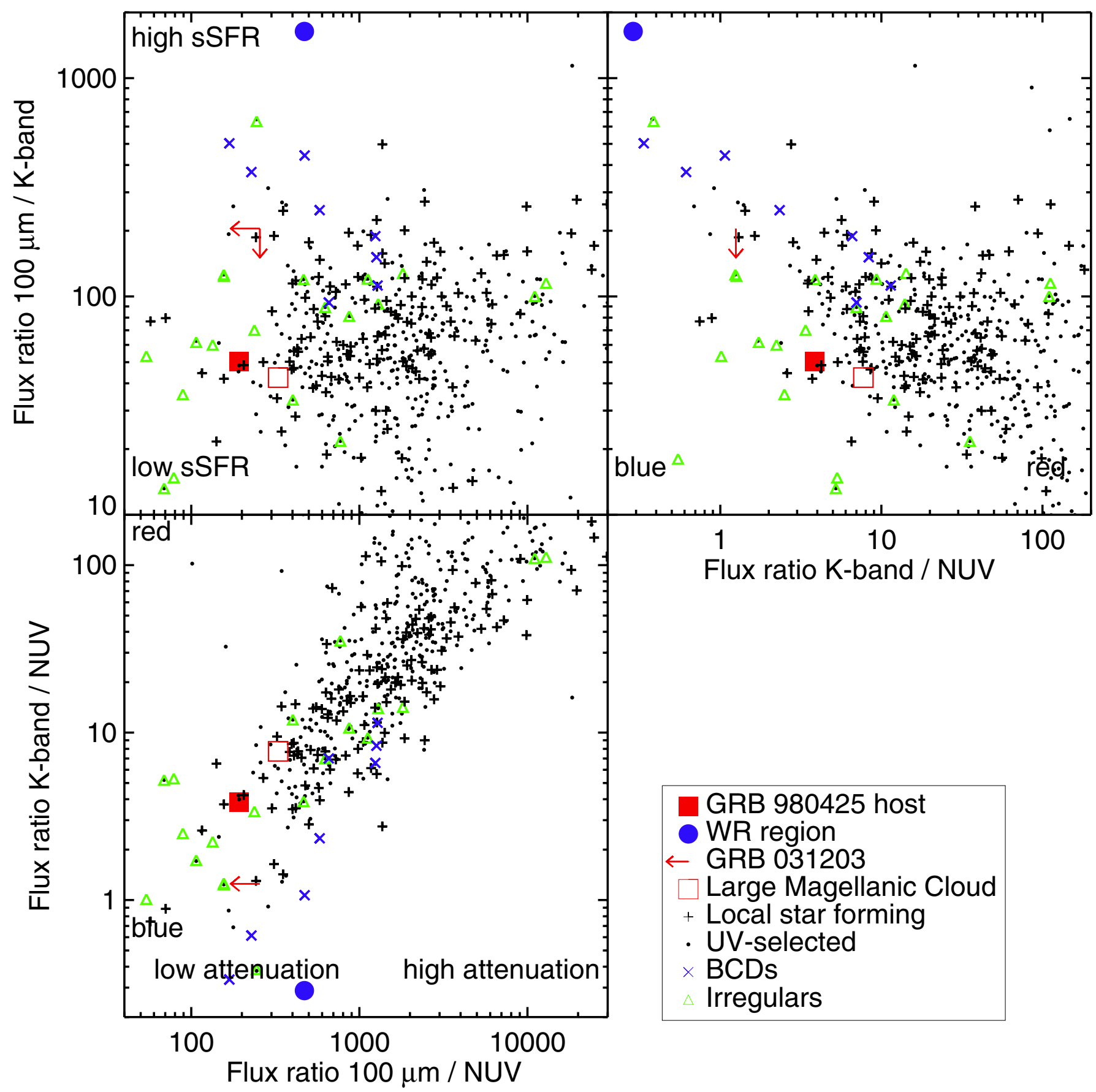

Fig. 3. Flux ratios of the GRB 980425 host (red filled square) and the WR region (blue circle) compared with GRB 031203 (red arrow; Watson et al. 2011), LMC (red open square; Israel et al. 2010; Meixner et al. 2013), local star-forming galaxies (plusses; Moustakas \& Kennicutt 2006; Kennicutt et al. 2011; Dale et al. 2007, 2012; Skibba et al. 2011), UV-selected galaxies (dots; Gil de Paz et al. 2007; irregular galaxies are marked as green triangles), irregular galaxies (triangles) and blue compact dwarfs ( $\times$; Hunt et al. 2005; Hirashita et al. 2008; Hunter et al. 2010). Low- $z$ GRB hosts exhibit low $100 \mu \mathrm{m} / \mathrm{NUV}$ flux ratios, which hints at low dust attenuation similar to that of irregular galaxies.

luminosity function of Sanders et al. (2003) shows that $90 \%$ of local galaxies are less luminous than the GRB 980425 host with $L_{\mathrm{IR}} \sim 10^{9} L_{\odot}$, and that $\sim 15 \%$ of star formation activity in the local universe happens in these faint galaxies. Therefore, assuming that the GRB rate traces the SFR density, it is not surprising to find a GRB in a dwarf galaxy.

\subsection{Dust and star formation in the WR region: high density}

The WR region is the most unusual feature of the GRB 980425 host, dominating its mid-IR, far-IR, and radio emission, and is the only star-forming region detected by ALMA at $2.8 \mathrm{~mm}$ (Figs. 1 and 2). We demonstrate here that its high ISM gas density can explain these properties, as it likely plays an important role in efficient dust production and efficient build-up of radio emission.

First, we checked whether its age is sufficient to form the dust that we observe in this object. Taking into account only the stars that can finish their main-sequence phase in $~ 10 \mathrm{Myr}$ (i.e. those with masses $>15 M_{\odot}$ ) we calculated an average dust yield per star in order to explain the dust mass we measured for the WR region (as in Michałowski et al. 2010c,b). Assuming 


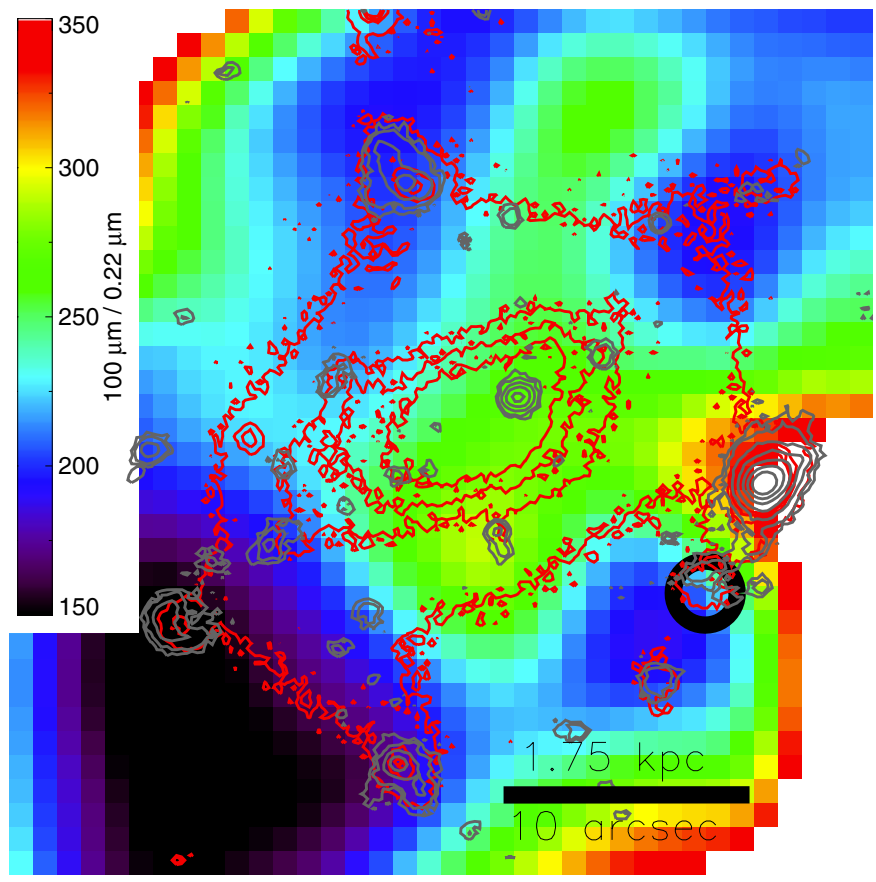

Fig. 4. $100 \mu \mathrm{m} / 0.22 \mu \mathrm{m}$ flux ratio map of the GRB 980425 host. The image is $35^{\prime \prime} \times 35^{\prime \prime}$ and is constructed from images adaptively smoothed to the same spatial scales using AsMOOTH (Ebeling et al. 2006) to avoid strong colour fluctuations. The contours represent the narrowband $\mathrm{H} \alpha$ map (grey, confirmed to be consistent with the IFU $\mathrm{H} \alpha$ image from Christensen et al. 2008) and $R$-band image (red; Sollerman et al. 2005). The black circle shows the GRB position. The WR region is to the north-west of this position. It is apparent that the low $100 \mu \mathrm{m} /$ NUV flux ratio (blue regions) corresponds to the spiral arms traced by star-forming regions (peaks of red and grey countours), with the exception of the WR region exhibiting very high $100 \mu \mathrm{m}$ flux

a Chabrier IMF, its stellar mass corresponds to 43000 of these stars. Hence, each of them should have produced $\sim 1.4 M_{\odot}$ of dust. This is close to the highest dust yields predicted theoretically by Nozawa et al. (2003, Fig. 12) and Gall et al. (2011, Fig. 6), and inferred observationally (Dunne et al. 2003, 2009b; Morgan et al. 2003; Gomez et al. 2009; Green et al. 2004; Ercolano et al. 2007; Meikle et al. 2007; Lee et al. 2009; Sibthorpe et al. 2010; Barlow et al. 2010). Given the possibility of rapid ( $10 \mathrm{Myr})$ dust grain growth in the ISM (Draine \& Salpeter 1979; Draine 1990, 2009; Hirashita 2000; Zhukovska et al. 2008; Michałowski et al. 2010b), which would lower the required dust yield per star, it is not unreasonable to assume that the dust present in the WR region could have been formed during a short ( $\sim 10 \mathrm{Myr})$ recent period of star formation, even if it had contained no pre-existing dust formed by AGB stars. Moreover, these massive stars would alone produce enough metals necessary for this dust production (not counting metals produced by older AGB stars). Indeed a SN produces $\lesssim 1 M_{\odot}$ of heavy elements (Todini \& Ferrara 2001; Nozawa et al. 2003; Bianchi \& Schneider 2007; Cherchneff \& Dwek 2009), which is close to what is required to explain the dust mass in the WR region.

The timescale of dust grain growth is inversely proportional to the gas density (Eq. (8) of Draine 2009) and is only 2 Myr at a density of $150 \mathrm{~cm}^{-3}$. Hence, the unusually high far-IR luminosity (Fig. 1) and dust mass of the WR region could be explained if it is the densest among the star-forming regions in the GRB 980425 host.

This is consistent with the finding of Christensen et al. (2008) who reported slightly higher electron density for this



Fig. 5. Dust-to-stellar mass ratio as a function of UV-to-optical colour of the GRB 980425 host (red square), LMC (open red square; $M_{*}$ from Skibba et al. 2012 and $M_{\mathrm{d}}$ derived applying the method of Bianchi 2013 to the data from Meixner et al. 2013), KINGFISH galaxies (blue plusses; Kennicutt et al. 2011), and the averages of other local galaxies in eight colour bins (Table 1 and Fig. 4 of Cortese et al. 2012). The solid line represents a linear fit to the data, which results in $M_{\mathrm{d}} / M_{*}=$ $-1.87 \pm 0.07$ at the host colour of NUV $-r \sim 1.5$, close to the measured value of $M_{\mathrm{d}} / M_{*} \sim-2.1$. The WR region is off this plot with NUV $-r \sim-0.15$ mag and $M_{\mathrm{d}} / M_{*} \sim-2.1$.

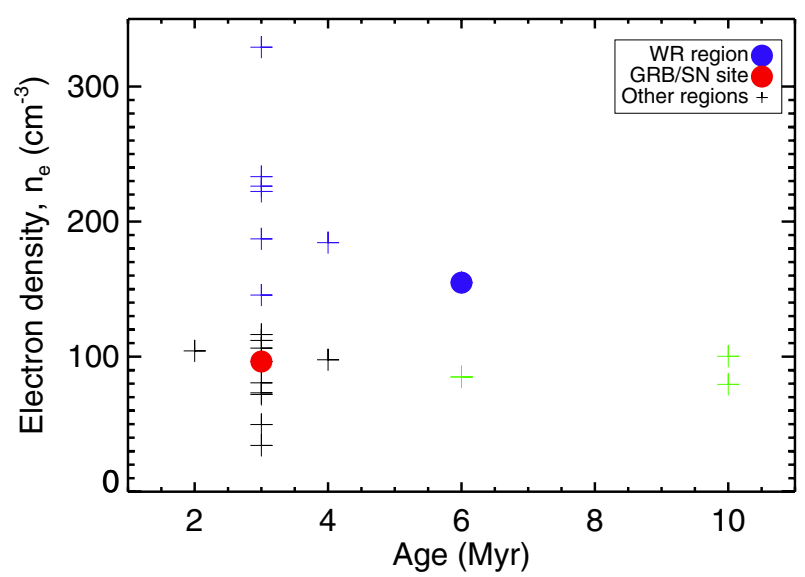

Fig. 6. Electron density of the star-forming regions in the GRB 980425 host (derived using the [S II] flux ratio from Christensen et al. 2008 using the calibration of O'Dell et al. 2013 and Osterbrock \& Ferland 2006) as a function of their ages from the SED modelling (Christensen et al. 2008). The WR region is both old and dense compared with other regions, which may have helped it to accumulate dust more efficiently. Blue and green crosses mark the regions with $n_{\mathrm{e}}>145 \mathrm{~cm}^{-3}$ and age $\geq 6 \mathrm{Myr}$, respectively (see also Fig. 1).

region compared with other star-forming regions. We recalculated the densities of all regions from the [S II] line ratios (Christensen et al. 2008) using the calibration of O'Dell et al. (2013) based on the model of Osterbrock \& Ferland (2006): $\log n_{\mathrm{e}}=4.705-1.9875[$ S II 6716]/[S II 6731]. We obtained $n_{\mathrm{e}}=150$ and $100 \mathrm{~cm}^{-3}$ for the WR region and the SN site, respectively (consistently with Christensen et al. 2008) and the mean for all regions of $130 \pm 15 \mathrm{~cm}^{-3}$. As shown in Fig. 1, five out of seven other regions with high densities $n_{\mathrm{e}}>145 \mathrm{~cm}^{-3}$ are located in the northern spiral arms, which is the third most prominent $100 \mu \mathrm{m}$ feature after the WR region and the central bar (the northernmost $100 \mu \mathrm{m}$ blob is not covered by the IFU data of Christensen et al. 2008). This supports the scenario in 


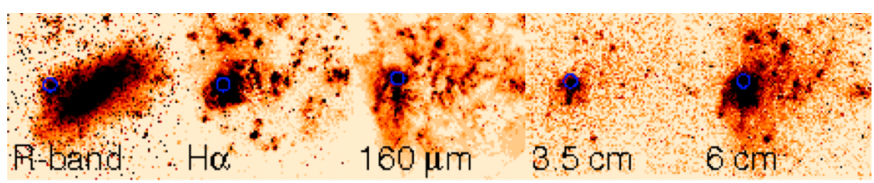

Fig. 7. Mosaic of the images of the Large Magellanic Cloud (Gaustad et al. 2001; Meixner et al. 2006; Dickel et al. 2005). North is up and east is to the left. Each panel is $6^{\circ} \times 6^{\circ}(4 \mathrm{kpc} \times 4 \mathrm{kpc})$. The blue circle show the position of the star-forming region 30 Doradus (Tarantula Nebula). The LMC with 30 Doradus dominating at $\mathrm{H} \alpha$, far-IR and radio is similar to the GRB 980425 host with the WR region (compare with Fig. 1).

which high density of the ISM is responsible for more efficient dust production.

These other star-forming regions with high $n_{\mathrm{e}}$ are all younger than the WR region (Fig. 6). This provides an explanation for why their $100 \mu \mathrm{m}$ emission is not as prominent as that of the WR region, as there was even less time to form dust. On the other hand, other regions (some older than the WR region) are not dense enough to accumulate dust efficiently. However, the regions older than the WR region (green crosses in Figs. 1 and 6) are all concentrated in the bar, which shows pronounced $100 \mu \mathrm{m}$ emission.

The high density of the WR region would also explain why it is the only region firmly detected at the radio continuum (Fig. 1). According to Hirashita \& Hunt (2006) the radio fluence of supernova remnants depends on gas density, naturally explaining the radio brightness of the WR region if it is dense. On the other hand, the other regions denser than the WR region are also younger (Fig. 6), so their synchrotron radiation might not have had time to build up yet. The WR region indeed exhibits a steep radio spectrum (Fig. 2 and Sect. 5.4), hinting at the synchrotron nature of the emission.

Finally, the ALMA detection of the WR region at $2.8 \mathrm{~mm}$ is also consistent with its high density. Free-free emission coefficients are proportional to the square of the density (Condon 1992) making the low density regions fainter than the WR. The dust-only emission is a $\sim 2.5 \sigma$ detection, so without the freefree emission ( $45 \%$ of the WR $2.8 \mathrm{~mm}$ emission) the WR region would probably not have been detected.

The WR region appears to be similar to the 30 Doradus (Tarantula Nebula), which also dominates the $\mathrm{H} \alpha$ (Gaustad et al. 2001), far-IR (Meixner et al. 2006), and radio (Dickel et al. 2005) emission of the LMC, as shown in Fig. 7, but it contributes very little to total stellar mass. The star-forming region 30 Doradus is very dense (with a density close to the value we measured for the WR region; e.g. Meixner et al. 2006; Pellegrini et al. 2010, 2011; Kawada et al. 2011; Pineda et al. 2012), which provides additional support for our hypothesis that the extreme properties of the WR region are due to its high density.

If high density environments are also found close to the positions of other GRBs, then the ISM density should also be considered, along with metallicity, an important factor influencing whether a given stellar population can produce a GRB. This is especially true if the Hammer et al. (2006) "runaway" hypothesis, that the GRB progenitor was born in the WR region and expelled from it, turns out to be correct. The properties of the WR region we discussed here (high SFR, high density, numerous massive stars, low age) are consistent with this hypothesis, but high-resolution observations of other GRB hosts are needed to test whether GRB progenitors are usually born in dense star clusters.

\subsection{Submillimetre excess and gas-to-dust ratio}

Many dwarf low-metallicity galaxies have been shown to exhibit a submillimetre excess, i.e. an enhancement in the submillimetre emission above the extrapolation from far-IR data (Lisenfeld et al. 2002; Galliano et al. 2003, 2005, 2011; Bendo et al. 2006; Galametz et al. 2009, 2011; Bot et al. 2010; Gordon et al. 2010; Israel et al. 2010; Planck Collaboration 2011a,b; Dale et al. 2012; De Looze et al. 2012). Apart from the cold dust emission, this excess has also been attributed to different grain properties (e.g. shallower spectral slope $\beta$ ), spinning dust, and magnetic nanograins (e.g. Reach et al. 1995; Draine \& Li 2007; Dale et al. 2012; Hermelo et al. 2013, and references therein).

The GRB 980425 host exhibits a $160 \mu \mathrm{m} / 870 \mu \mathrm{m}$ flux ratio of $\sim 25$, lower than the lowest values found by Galametz et al. (2011) for local dwarfs, indicating a very strong submillimetre excess. This is not because of the synchrotron contribution to the submillimetre flux, which should be minimal given the flux of $0.42 \mathrm{mJy}$ at $6 \mathrm{~cm}$ and a negative radio slope (see Sect. 5.4 and Michałowski et al. 2009), nor is it due to contamination by free-free emission because, as we found in Sect. 3, it contributes at most $2 \%$ to the total $870 \mu \mathrm{m}$ flux.

Given the far-IR wavelength coverage, we are in a position to investigate the submillimetre excess in the GRB 980425 host. We present two hypotheses to explain this excess: very cold dust and unusual dust properties. Under the assumption of normal dust properties, based on the SED fit (Fig. 2) we predict an $870 \mu \mathrm{m}$ flux of $\sim 7.5 \mathrm{mJy}$. This is about half the measured value of $\sim 13 \mathrm{mJy}$ (Table 1 ). If this excess is interpreted as a contribution of very cold $(10 \mathrm{~K})$ dust, not accounted for in our SED modelling, then it transforms into an additional $2 \times$ $10^{6} M_{\odot}$ of dust (with $\beta=1.5$ ), doubling the dust content in the GRB 980425 host.

Without this cold dust, its molecular gas-to-dust ratio is $M_{\mathrm{H}_{2}} / M_{\mathrm{d}}<185$ using the $\mathrm{H}_{2}$ gas mass upper limit from Hatsukade et al. (2007). This is consistent with that of the Milky Way ( 100-400; Sodroski et al. 1997; Draine et al. 2007) and other spirals ( 100-1000; Devereux \& Young 1990; Stevens et al. 2005). However, if the submillimetre excess is indeed due to cold dust, then the limit for the GRB 980425 host lowers to $M_{\mathrm{H}_{2}} / M_{\mathrm{d}}<82$. This indicates much higher dust content, consistent with that of high-redshift submillimetre galaxies $(\sim 50$; Kovács et al. 2006; Michałowski et al. 2010c), of the nuclear regions of local (ultra)luminous IR galaxies (120 \pm 28 ; Wilson et al. 2008) and of local, far-IR-selected galaxies ( 50; Seaquist et al. 2004). This can be confirmed by a detection of a CO line of the GRB 980425 host to constrain the molecular gas content, and by investigation of the submillimetre excess at other wavelengths and at higher resolution, allowing a more precise study of its origin. This is now possible with the current capabilities of ALMA.

The additional cold dust is not the only possible explanation of the submillimetre excess in the GRB 980425 host. As indicated in Sect. 4.1, we are able to fit all far-IR and submillimetre data with a modified black-body curve with a shallow spectral slope of $\beta=1$ without the need to invoke cold dust (see Fig. 13 of Bendo et al. 2006 for a similar result for another galaxy). In this scenario, a very low $160 \mu \mathrm{m} / 870 \mu \mathrm{m}$ flux ratio of the GRB 980425 host would be a result of unusual dust properties favouring emission at longer wavelengths. Alternatively, such a shallow slope could indicate dust heating by an unusually low-intensity radiation field which results in a wider range of dust temperatures along the line of sight (Hunt et al. 2014b). 
Our low-resolution $870 \mu \mathrm{m}$ data do not allow the study of the spatial distribution of the submillimetre excess. However, ALMA data provide a hint that the WR region may be responsible for the excess. Nevertheless, because almost half of the observed $2.8 \mathrm{~mm}$ flux is free-free emission, sensitive highresolution submillimetre observations, probing cold dust emission, are necessary to confirm this.

\subsection{Infrared-radio correlation}

Based on the extrapolation from the $24 \mu \mathrm{m}$ data, Michałowski et al. (2009) suggested that the radio emission of the GRB 980425 host is much lower than expected from the infrared-radio correlation (Condon 1992). However, with the far-IR wavelength coverage provided by our Herschel data we revised the infrared luminosity estimate to be a factor of $\sim 2.5$ lower. In order to quantify the offset of the host from the infrared-radio correlation, we calculated the parameter $q=\log \left(L_{\mathrm{IR}}\left[L_{\odot}\right] / 3.75 \times 10^{12} / L_{\nu 1.4 \mathrm{GHz}}\left[L_{\odot} \mathrm{Hz}^{-1}\right]\right)$, where $L_{v 1.4 \mathrm{GHz}}$ is the rest-frame $1.4 \mathrm{GHz}$ luminosity density computed from the power-law fit to our radio data and the $4.8 \mathrm{GHz}$ data of Michałowski et al. (2009). We find a spectral index of $\alpha=-0.62 \pm 0.15$ (consistent with a typical value of -0.75 for other star-forming galaxies Condon 1992; Clemens et al. 2008; Dunne et al. 2009a; Ibar et al. 2010) and a rest-frame $1.4 \mathrm{GHz}$ flux of $0.91 \pm 0.17 \mathrm{mJy}$, which corresponds to a $L_{\gamma}$ rest $1.4 \mathrm{GHz}=$ $1.5 \times 10^{27} \mathrm{erg} \mathrm{s}^{-1} \mathrm{~Hz}^{-1}$. This translates to $q=2.8 \pm 0.5$, fully consistent with the average value for local star-forming galaxies of 2.64 (with a scatter of 0.26; Bell 2003).

In a similar way, for the WR region we derived a steeper $\alpha=-1.08 \pm 0.18, F_{v \text { rest } 1.4 \mathrm{GHz}}=0.56 \pm 0.08, L_{v \text { rest } 1.4 \mathrm{GHz}}=$ $8.9 \times 10^{26} \mathrm{erg} \mathrm{s}^{-1} \mathrm{~Hz}^{-1}$, and $q=2.48 \pm 0.37$. This $q$ value is also consistent with that of local galaxies within the errors. A slightly lower $q$ value may indicate that the radio emission in the WR region is building up faster than the dust content.

The mid-infrared and radio (close to the rest-frame $1.4 \mathrm{GHz}$ ) data presented by Watson et al. (2011) for the GRB 031203 host $\left(L_{\mathrm{IR}} \sim 2 \times 10^{10} L_{\odot}, L_{v 1.4 \mathrm{GHz}} \sim 10^{29} \mathrm{erg} \mathrm{s}^{-1} \mathrm{~Hz}^{-1}\right)$ also implies a low $q=2.25$. However, the data of Watson et al. (2011) extends only up to $30 \mu \mathrm{m}$, so increased wavelength coverage is required to confirm this result.

\section{Conclusions}

Using high-resolution observations from Herschel, APEX, ALMA, and ATCA we investigated the properties of the ISM in the GRB 980425 host, and we found that it is characterised by relatively low dust content and a high fraction of UV-visible star formation, similar to other dwarf galaxies. These galaxies are abundant in the local universe, so it is not surprising to find a GRB in one of them, assuming the correspondence between the GRB rate and star formation.

The star-forming region displaying the Wolf-Rayet signatures in the spectrum (WR region), located $800 \mathrm{pc}$ from the GRB position, contributes substantially to the host emission at the far-infrared, millimetre, and radio wavelengths and we propose this to be a consequence of its high gas density. If dense environments are also found close to the positions of other GRBs, then the ISM density should also be considered, along with metallicity, as an important factor influencing whether a given stellar population can produce a GRB, especially if it turns out that GRB progenitors in general (and that of GRB 980425 in particular) are born in dense star clusters. The brightness of the
WR region also indicates that it may be responsible for the submillimetre excess we detected for the host.

Acknowledgements. We thank Joanna Baradziej for help with improving this paper; Tom Muxlow, Eelco van Kampen, and Robert Braun for help with the ALMA and ATCA observations; Lise Christensen for kindly providing the IFU maps from Christensen et al. (2008); our anonymous referee, Vincent HénaultBrunet, and Françoise Combes for useful comments; and Elisabete da Cunha for providing updated filter profiles for MAGPHYS. M.J.M. and G.G. are postdoctoral researchers of the FWO-Vlaanderen (Belgium). M.J.M. acknowledges the support of the Science and Technology Facilities Council. L.K.H. and S.B. are supported by the INAF PRIN 2012 grant. The Dark Cosmology Centre is funded by the Danish National Research Foundation. T.M. and D.B. acknowledge the support of the Australian Research Council through grant DP110102034. AdUP acknowledges support from the European Commission (FP7-PEOPLE-2012CIG 322307) and from the Spanish project AYA2012-39362-C02-02. PACS has been developed by a consortium of institutes led by MPE (Germany) and including UVIE (Austria); KU Leuven, CSL, IMEC (Belgium); CEA, LAM (France); MPIA (Germany); INAF-IFSI/OAA/OAP/OAT, LENS, SISSA (Italy); IAC (Spain). This development has been supported by the funding agencies BMVIT (Austria), ESA-PRODEX (Belgium), CEA/CNES (France), DLR (Germany), ASI/INAF (Italy), and CICYT/MCYT (Spain). SPIRE has been developed by a consortium of institutes led by Cardiff University (UK) and including Univ. Lethbridge (Canada); NAOC (China); CEA, LAM (France); IFSI, Univ. Padua (Italy); IAC (Spain); Stockholm Observatory (Sweden); Imperial College London, RAL, UCL-MSSL, UKATC, Univ. Sussex (UK); and Caltech, JPL, NHSC, Univ. Colorado (USA). This development has been supported by national funding agencies: CSA (Canada); NAOC (China); CEA, CNES, CNRS (France); ASI (Italy); MCINN (Spain); SNSB (Sweden); STFC (UK); and NASA (USA). This paper makes use of the following ALMA data: ADS/JAO.ALMA\#2011.0.00046.S. ALMA is a partnership of ESO (representing its member states), NSF (USA) and NINS (Japan), together with NRC (Canada) and NSC and ASIAA (Taiwan), in cooperation with the Republic of Chile. The Joint ALMA Observatory is operated by ESO, AUI/NRAO and NAOJ. This publication is based on data acquired with the Atacama Pathfinder Experiment (APEX). APEX is a collaboration between the Max-Planck-Institut fur Radioastronomie, the European Southern Observatory, and the Onsala Space Observatory. The Australia Telescope is funded by the Commonwealth of Australia for operation as a National Facility managed by CSIRO. This research has made use of data obtained from the High Energy Astrophysics Science Archive Research Center (HEASARC), provided by NASA's Goddard Space Flight Center. This publication makes use of data products from the Widefield Infrared Survey Explorer, which is a joint project of the University of California, Los Angeles, and the Jet Propulsion Laboratory/California Institute of Technology, funded by the National Aeronautics and Space Administration. This research has made use of the GHostS database (http://www.grbhosts . org), which is partly funded by Spitzer/NASA grant RSA Agreement No. 1287913; the NASA/IPAC Extragalactic Database (NED) which is operated by the Jet Propulsion Laboratory, California Institute of Technology, under contract with the National Aeronautics and Space Administration; SAOImage DS9, developed by Smithsonian Astrophysical Observatory (Joye \& Mandel 2003); and the NASA's Astrophysics Data System Bibliographic Services.

\section{References}

Barlow, M. J., Krause, O., Swinyard, B. M., et al. 2010, A\&A, 518, L138 Bell, E. F. 2003, ApJ, 586, 794

Bendo, G. J., Dale, D. A., Draine, B. T., et al. 2006, ApJ, 652, 283 Berger, E., Cowie, L. L., Kulkarni, S. R., et al. 2003, ApJ, 588, 99 Bianchi, S. 2013, A\&A, 552, A89

Bianchi, S., \& Schneider, R. 2007, MNRAS, 378, 973

Blaauw, A. 1993, in Massive Stars: Their Lives in the Interstellar Medium, eds.

J. P. Cassinelli, \& E. B. Churchwell, ASP Conf. Ser., 35, 207

Bloom, J. S., Kulkarni, S. R., \& Djorgovski, S. G. 2002, AJ, 123, 1111

Boissier, S., Salvaterra, R., Le Floc'h, E., et al. 2013, A\&A, 557, A34

Boquien, M., Calzetti, D., Combes, F., et al. 2011, AJ, 142, 111

Boquien, M., Buat, V., Boselli, A., et al. 2012, A\&A, 539, A145

Bot, C., Ysard, N., Paradis, D., et al. 2010, A\&A, 523, A20

Bruzual, G., \& Charlot, S. 2003, MNRAS, 344, 1000

Caplan, J., \& Deharveng, L. 1986, A\&A, 155, 297

Cardelli, J. A., Clayton, G. C., \& Mathis, J. S. 1989, ApJ, 345, 245

Castro Cerón, J. M., Michałowski, M. J., Hjorth, J., et al. 2006, ApJ, 653, L85

Castro Cerón, J. M., Michałowski, M. J., Hjorth, J., et al. 2010, ApJ, 721, 1919

Chabrier, G. 2003, ApJ, 586, L133

Chanial, P., Flores, H., Guiderdoni, B., et al. 2007, A\&A, 462, 81 
Chen, H. W., Prochaska, J. X., Ramirez-Ruiz, E., et al. 2007, ApJ, 663, 420 Cherchneff, I., \& Dwek, E. 2009, ApJ, 703, 642

Christensen, L., Vreeswijk, P. M., Sollerman, J., et al. 2008, A\&A, 490, 45 Ciesla, L., Boselli, A., Smith, M. W. L., et al. 2012, A\&A, 543, A161 Clemens, M. S., Vega, O., Bressan, A., et al. 2008, A\&A, 477, 95 Condon, J. J. 1992, ARA\&A, 30, 575

Conselice, C. J., Vreeswijk, P. M., Fruchter, A. S., et al. 2005, ApJ, 633, 29 Cortese, L., Ciesla, L., Boselli, A., et al. 2012, A\&A, 540, A52 da Cunha, E., Charlot, S., \& Elbaz, D. 2008, MNRAS, 388, 1595 da Cunha, E., Eminian, C., Charlot, S., \& Blaizot, J. 2010, MNRAS, 403, 1894 Dale, D. A., Gil de Paz, A., Gordon, K. D., et al. 2007, ApJ, 655, 863 Dale, D. A., Cohen, S. A., Johnson, L. C., et al. 2009, ApJ, 703, 517 Dale, D. A., Aniano, G., Engelbracht, C. W., et al. 2012, ApJ, 745, 95 De Looze, I., Baes, M., Parkin, T. J., et al. 2012, MNRAS, 423, 2359 de Ugarte Postigo, A., Lundgren, A., Martín, S., et al. 2012, A\&A, 538, A44 Devereux, N. A., \& Young, J. S. 1990, ApJ, 359, 42

de Wit, W. J., Testi, L., Palla, F., \& Zinnecker, H. 2005, A\&A, 437, 247

Dickel, J. R., McIntyre, V. J., Gruendl, R. A., \& Milne, D. K., 2005, AJ, 129, 790

Draine, B. T. 1990, in The Evolution of the Interstellar Medium, ed. L. Blitz, ASP Conf. Ser., 12, 193

Draine, B. T. 2009, in ASP Conf. Ser. 414, eds. T. Henning, E. Grün, \& J. Steinacker, 453

Draine, B. T., \& Li, A. 2007, ApJ, 657, 810

Draine, B. T., \& Salpeter, E. E. 1979, ApJ, 231, 438

Draine, B. T., Dale, D. A., Bendo, G., et al. 2007, ApJ, 663, 866

Dunne, L., Eales, S., Edmunds, M., et al. 2000, MNRAS, 315, 115

Dunne, L., Eales, S., Ivison, R., Morgan, H., \& Edmunds, M. 2003, Nature, 424, 285

Dunne, L., Ivison, R. J., Maddox, S., et al. 2009a, MNRAS, 394, 3

Dunne, L., Maddox, S. J., Ivison, R. J., et al. 2009b, MNRAS, 394, 1307

Ebeling, H., White, D. A., \& Rangarajan, F. V. N. 2006, MNRAS, 368, 65 Elliott, J., Greiner, J., Khochfar, S., et al. 2012, A\&A, 539, A113

Engelbracht, C. W., Rieke, G. H., Gordon, K. D., et al. 2008, ApJ, 678, 804 Ercolano, B., Barlow, M. J., \& Sugerman, B. E. K. 2007, MNRAS, 375, 753 Foley, S., Watson, D., Gorosabel, J., et al. 2006, A\&A, 447, 891

Frail, D. A., Bertoldi, F., Moriarty-Schieven, G. H., et al. 2002, ApJ, 565, 829 Fruchter, A. S., Levan, A. J., Strolger, L., et al. 2006, Nature, 441, 463 Fynbo, J. P. U., Holland, S., Andersen, M. I., et al. 2000, ApJ, 542, L89 Fynbo, J. P. U., Jakobsson, P., Prochaska, J. X., et al. 2009, ApJS, 185, 526 Galama, T. J., Vreeswijk, P. M., van Paradijs, J., et al. 1998, Nature, 395, 670 Galametz, M., Madden, S., Galliano, F., et al. 2009, A\&A, 508, 645 Galametz, M., Madden, S. C., Galliano, F., et al. 2011, A\&A, 532, A56 Gall, C., Hjorth, J., \& Andersen, A. C. 2011, A\&ARv, 19, 43

Galliano, F., Madden, S. C., Jones, A. P., et al. 2003, A\&A, 407, 159

Galliano, F., Madden, S. C., Jones, A. P., Wilson, C. D., \& Bernard, J. P. 2005, A\&A, 434, 867

Galliano, F., Hony, S., Bernard, J. P., et al. 2011, A\&A, 536, A88

Gaustad, J. E., McCullough, P. R., Rosing, W., \& Van Buren, D. 2001, PASP, 113,1326

Gavazzi, G., Fumagalli, M., Fossati, M., et al. 2013, A\&A, 553, A89 Gehrels, N., Chincarini, G., Giommi, P., et al. 2004, ApJ, 611, 1005 Gil de Paz, A., Boissier, S., Madore, B. F., et al. 2007, ApJS, 173, 185 Gilbank, D. G., Bower, R. G., Glazebrook, K., et al. 2011, MNRAS, 414, 304 Gomez, H. L., Dunne, L., Ivison, R. J., et al. 2009, MNRAS, 397, 1621 Gordon, K. D., Galliano, F., Hony, S., et al. 2010, A\&A, 518, L89 Green, D. A., Tuffs, R. J., \& Popescu, C. C. 2004, MNRAS, 355, 1315 Griffin, M. J., Abergel, A., Abreu, A., et al. 2010, A\&A, 518, L3 Güsten, R., Nyman, L.Å., Schilke, P., et al. 2006, A\&A, 454, L13 Hammer, F., Flores, H., Schaerer, D., et al. 2006, A\&A, 454, 103 Han, X. H., Hammer, F., Liang, Y. C., et al. 2010, A\&A, 514, A24 Hao, J. M., \& Yuan, Y. F. 2013, ApJ, 772, 42

Hatsukade, B., Kohno, K., Endo, A., et al. 2007, PASJ, 59, 67

Hermelo, I., Lisenfeld, U., Relaño, M., et al. 2013, A\&A, 549, A70

Hirashita, H. 2000, PASJ, 52, 585

Hirashita, H., \& Hunt, L. K. 2006, A\&A, 460, 67

Hirashita, H., Kaneda, H., Onaka, T., \& Suzuki, T. 2008, PASJ, 60, 477

Hjorth, J., \& Bloom, J. S. 2012, The Gamma-Ray Burst - Supernova Connection, eds. C. Kouveliotou, R. A. M. J. Wijers, \& S. E. Woosley (Cambridge University Press), 169

Hjorth, J., Sollerman, J., Møller, P., et al. 2003, Nature, 423, 847

Hjorth, J., Malesani, D., Jakobsson, P., et al. 2012, ApJ, 756, 187

Hunt, L., Bianchi, S., \& Maiolino, R. 2005, A\&A, 434, 849

Hunt, L., Magrini, L., Galli, D., et al. 2012, MNRAS, 427, 906

Hunt, L. K., Palazzi, E., Michałowski, M. J., et al. 2014a, A\&A, submitted

Hunt, L. K., Testi, L., Casasola, V., et al. 2014b, A\&A, 561, A49

Hunter, D. A., Elmegreen, B. G., \& Ludka, B. C. 2010, AJ, 139, 447

Ibar, E., Ivison, R. J., Best, P. N., et al. 2010, MNRAS, 401, L53
Iglesias-Páramo, J., Buat, V., Hernández-Fernández, J., et al. 2007, ApJ, 670, 279

Israel, F. P., Wall, W. F., Raban, D., et al. 2010, A\&A, 519, A67

Jarrett, T. H., Chester, T., Cutri, R., Schneider, S. E., \& Huchra, J. P. 2003, AJ, 125,525

Joye, W. A., \& Mandel, E. 2003, in Astronomical Data Analysis Software and Systems XII, eds. H. E. Payne, R. I. Jedrzejewski, \& R. N. Hook, ASP Conf. Ser., 295, 489

Kawada, M., Takahashi, A., Yasuda, A., et al. 2011, PASJ, 63, 903

Kelly, P. L., Kirshner, R. P., \& Pahre, M. 2008, ApJ, 687, 1201

Kennicutt, R. C. 1998, ARA\&A, 36, 189

Kennicutt, R. C., Calzetti, D., Aniano, G., et al. 2011, PASP, 123, 1347

Kewley, L. J., \& Ellison, S. L. 2008, ApJ, 681, 1183

Kinney, A. L., Bohlin, R. C., Calzetti, D., Panagia, N., \& Wyse, R. F. G. 1993, ApJS, 86, 5

Kistler, M. D., Yüksel, H., Beacom, J. F., Hopkins, A. M., \& Wyithe, J. S. B. 2009, ApJ, 705, L104

Kovács, A. 2008, in SPIE Conf. Ser., 7020, 45

Kovács, A., Chapman, S. C., Dowell, C. D., et al. 2006, ApJ, 650, 592

Le Floc'h, E., Charmandaris, V., Forrest, W. J., et al. 2006, ApJ, 642, 636

Le Floc'h, E., Charmandaris, V., Gordon, K., et al. 2012, ApJ, 746, 7

Lee, H., Koo, B., Moon, D., et al. 2009, ApJ, 706, 441

Leloudas, G., Sollerman, J., Levan, A. J., et al. 2010, A\&A, 518, A29

Leloudas, G., Gallazzi, A., Sollerman, J., et al. 2011, A\&A, 530, A95

Levesque, E. M., Berger, E., Kewley, L. J., \& Bagley, M. M. 2010a, AJ, 139, 694

Levesque, E. M., Kewley, L. J., Berger, E., \& Jabran Zahid, H. 2010b, AJ, 140, 1557

Levesque, E. M., Berger, E., Soderberg, A. M., \& Chornock, R. 2011, ApJ, 739, 23

Lisenfeld, U., Israel, F. P., Stil, J. M., \& Sievers, A. 2002, A\&A, 382, 860

Lisenfeld, U., Verdes-Montenegro, L., Sulentic, J., et al. 2007, A\&A, 462, 507

McMullin, J. P., Waters, B., Schiebel, D., Young, W., \& Golap K. 2007, in Astronomical Data Analysis Software and Systems XVI, eds. R. A. Shaw, F. Hill, \& D. J. Bell, ASP Conf. Ser., 376, 127

Meikle, W. P. S., Mattila, S., Pastorello, A., et al. 2007, ApJ, 665, 608

Meixner, M., Gordon, K. D., Indebetouw, R., et al. 2006, AJ, 132, 2268

Meixner, M., Panuzzo, P., Roman-Duval, J., et al. 2013, AJ, 146, 62

Michałowski, M. J., Hjorth, J., Castro Cerón, J. M., \& Watson, D. 2008, ApJ, 672,817

Michałowski, M. J., Hjorth, J., Malesani, D., et al. 2009, ApJ, 693, 347

Michałowski, M., Hjorth, J., \& Watson, D. 2010a, A\&A, 514, A67

Michałowski, M. J., Murphy, E. J., Hjorth, J., et al. 2010b, A\&A, 522, A15

Michałowski, M. J., Watson, D., \& Hjorth, J. 2010c, ApJ, 712, 942

Michałowski, M. J., Dunlop, J. S., Cirasuolo, M., et al. 2012a, A\&A, 541, A85

Michałowski, M. J., Kamble, A., Hjorth, J., et al. 2012b, ApJ, 755, 85

Morgan, H. L., Dunne, L., Eales, S. A., Ivison, R. J., \& Edmunds, M. G. 2003, ApJ, 597, L33

Moustakas, J., \& Kennicutt, R. C. Jr. 2006, ApJS, 164, 81

Nguyen, H. T., Schulz, B., Levenson, L., et al. 2010, A\&A, 518, L5

Nozawa, T., Kozasa, T., Umeda, H., Maeda, K., \& Nomoto, K. 2003, ApJ, 598, 785

O’Dell, C. R., Ferland, G. J., Henney, W. J., \& Peimbert, M. 2013, AJ, 145, 93

O'Donnell, J. E. 1994, ApJ, 422, 158

Osterbrock, D. E., \& Ferland, G. J. 2006, Astrophysics of gaseous nebulae and active galactic nuclei, 2nd edn; Mill Valley (CA: Univ. Science Books)

Östlin, G., Zackrisson, E., Sollerman, J., Mattila, S., \& Hayes, M. 2008, MNRAS, 387, 1227

Ott, S. 2010, in Astronomical Data Analysis Software and Systems XIX, eds. Y. Mizumoto, K. I. Morita, \& M. Ohishi, ASP Conf. Ser., 434, 139

Pellegrini, E. W., Baldwin, J. A., \& Ferland, G. J. 2010, ApJS, 191, 160

Pellegrini, E. W., Baldwin, J. A., \& Ferland, G. J. 2011, ApJ, 738, 34

Perley, D. A., Levan, A. J., Tanvir, N. R., et al. 2013, ApJ, 778, 128

Pilbratt, G. L., Riedinger, J. R., Passvogel, T., et al. 2010, A\&A, 518, L1

Pineda, J. L., Mizuno, N., Röllig, M., et al. 2012, A\&A, 544, A84

Planck Collaboration 2011a, A\&A, 536, A17

Planck Collaboration 2011b, A\&A, 536, A16

Poglitsch, A., Waelkens, C., Geis, N., et al. 2010, A\&A, 518, L2

Reach, W. T., Dwek, E., Fixsen, D. J., et al. 1995, ApJ, 451, 188

Rice, W., Lonsdale, C. J., Soifer, B. T., et al. 1988, ApJS, 68, 91

Robertson, B. E., \& Ellis, R. S. 2012, ApJ, 744, 95

Roming, P. W. A., Kennedy, T. E., Mason, K. O., et al. 2005, Space Sci. Rev., 120,95

Roussel, H. 2013, PASP, 125, 1126

Sanders, D. B., Mazzarella, J. M., Kim, D. C., Surace, J. A., \& Soifer, B. T. 2003, AJ, 126, 1607 
M. J. Michałowski et al.: Spatially-resolved dust properties of the GRB 980425 host galaxy

Sault, R. J, \& Killeen, N. E. B. 2004, The Miriad User's Guide. Australia Telescope National Facility, Sydney (http://www.atnf.csiro. au/computing/software/miriad/)

Sault, R. J., Teuben, P. J., \& Wright, M. C. H. 1995, in Astronomical Data Analysis Software and Systems IV, eds. R. A. Shaw, H. E. Payne, \& J. J. E. Hayes, ASP Conf. Ser., 77, 433

Savaglio, S., Glazebrook, K., \& LeBorgne, D. 2009, ApJ, 691, 182

Schlegel, D. J., Finkbeiner, D. P., \& Davis, M. 1998, ApJ, 500, 525

Schulze, S., Klose, S., Björnsson, G., et al. 2011, A\&A, 526, A23

Seaquist, E., Yao, L., Dunne, L., \& Cameron, H. 2004, MNRAS, 349, 1428

Sibthorpe, B., Ade, P. A. R., Bock, J. J., et al. 2010, ApJ, 719, 1553

Silva, L., Granato, G. L., Bressan, A., \& Danese, L. 1998, ApJ, 509, 103

Siringo, G., Kreysa, E., Kovács, A., et al. 2009, A\&A, 497, 945

Skibba, R. A., Engelbracht, C. W., Dale, D., et al. 2011, ApJ, 738, 89

Skibba, R. A., Engelbracht, C. W., Aniano, G., et al. 2012, ApJ, 761, 42

Sodroski, T. J., Odegard, N., Arendt, R. G., et al. 1997, ApJ, 480, 173

Soifer, B. T., Boehmer, L., Neugebauer, G., \& Sanders, D. B. 1989, AJ, 98, 766

Sollerman, J., Holland, S. T., Challis, P., et al. 2002, A\&A, 386, 944

Sollerman, J., Östlin, G., Fynbo, J. P. U., et al. 2005, New Astron., 11, 103

Stanek, K. Z., Matheson, T., Garnavich, P. M., et al. 2003, ApJ, 591, L17

Stanek, K. Z., Gnedin, O. Y., Beacom, J. F., et al. 2006, Acta Astron., 56, 333

Stevens, J. A., Amure, M., \& Gear, W. K. 2005, MNRAS, 357, 361
Svensson, K. M., Levan, A. J., Tanvir, N. R., Fruchter, A. S., \& Strolger, L. 2010, MNRAS, 405, 57

Tanvir, N. R., Barnard, V. E., Blain, A. W., et al. 2004, MNRAS, 352, 1073

Tenjes, P., Einasto, J., Maitzen, H. M., \& Zinnecker, H. 2001, A\&A, 369, 530

Thöne, C. C., Fynbo, J. P. U., Östlin, G., et al. 2008, ApJ, 676, 1151

Thuan, T. X. 1983, ApJ, 268, 667

Tinney, C., Stathakis, R., Cannon, R., et al. 1998, IAU Circ., 6896

Todini, P., \& Ferrara, A. 2001, MNRAS, 325, 726

Wainwright, C., Berger, E., \& Penprase, B. E. 2007, ApJ, 657, 367

Wang, W. H., Chen, H. W., \& Huang, K. Y. 2012, ApJ, 761, L32

Watson, D., Hjorth, J., Jakobsson, P., et al. 2004, A\&A, 425, L33

Watson, D., French, J., Christensen, L., et al. 2011, ApJ, 741, 58

Wilson, C. D., Petitpas, G. R., Iono, D., et al. 2008, ApJS, 178, 189

Woo, J., Courteau, S., \& Dekel, A. 2008, MNRAS, 390, 1453

Woosley, S. E. 1993, ApJ, 405, 273

Woosley, S. E., \& Heger, A. 2006, ApJ, 637, 914

Wright, E. L., Eisenhardt, P. R. M., Mainzer, A. K., et al. 2010, AJ, 140, 1868

Yoon, S. C., \& Langer, N. 2005, A\&A, 443, 643

Yoon, S. C., Langer, N., \& Norman, C. 2006, A\&A, 460, 199

Yüksel, H., Kistler, M. D., Beacom, J. F., \& Hopkins, A. M. 2008, ApJ, 683, L5

Zhukovska, S., Gail, H. P., \& Trieloff, M. 2008, A\&A, 479, 453 